\title{
On Achievable Rates and Complexity of LDPC Codes over Parallel Channels: Bounds and Applications
}

\author{
Igal Sason, Member and Gil Wiechman, Graduate Student Member
}

\begin{abstract}
A variety of communication scenarios can be modeled by a set of parallel channels. Upper bounds on the achievable rates under maximum-likelihood decoding, and lower bounds on the decoding complexity per iteration of ensembles of lowdensity parity-check (LDPC) codes are presented. The communication of these codes is assumed to take place over statistically independent parallel channels where the component channels are memoryless, binary-input and output-symmetric. The bounds are applied to ensembles of punctured LDPC codes where the puncturing patterns are either random or possess some structure. Our discussion is concluded by a diagram showing interconnections between the new theorems and some previously reported results.
\end{abstract}

\section{Index Terms}

Complexity, low-density parity-check (LDPC) codes, maximum-likelihood (ML) decoding, iterative decoding, parallel channels, punctured codes.

\section{INTRODUCTION}

Parallel channels serve as a model for analyzing various communication scenarios, e.g., rate-compatible puncturing of error-correcting codes, non-uniformly error-protected codes, transmission over block-fading channels and multicarrier signaling. All these scenarios can be modeled as a transmission of information over a set of parallel channels where each code symbol is assigned to one of these component channels. Naturally, analytical tools for evaluating the performance and decoding complexity of error-correcting codes whose transmission takes place over a set of parallel channels are gaining theoretical and practical interest (see, e.g., [3], [7], [15]).

The channel model considered in this paper assumes that the communication of binary linear block codes takes place over $J$ statistically independent component channels where each of the individual channels is a memoryless binary-input output-symmetric (MBIOS) channel whose probability density function is given by $p(\cdot \mid \cdot ; j)(j=$ $1,2, \ldots, J)$. If we let $\mathcal{I}(j)$ denote the set of indices of the symbols in an $n$-length codeword which are transmitted over the $j^{\text {th }}$ channel, then

$$
p_{n}(\underline{y} \mid \underline{x})=\prod_{j=1}^{J} \prod_{i \in \mathcal{I}(j)} p\left(y_{i} \mid x_{i} ; j\right) .
$$

This paper focuses primarily on information-theoretic aspects of low-density parity-check (LDPC) codes whose transmission takes place over a set of parallel channels. It provides upper bounds on the achievable rates under maximum-likelihood (ML) decoding, and lower bounds on the decoding complexity per iteration of ensembles of LDPC codes. The paper forms a generalization of the results in [17]. However, the bounds on the achievable rates and decoding complexity derived in this paper hold asymptotically with probability 1 for ensembles of LDPC codes where we let their block length tend to infinity; this is in contrast to the results in [17] which refer to communication over a single MBIOS channel, and are valid code by code. The bounds introduced in this paper are applied to ensembles of punctured LDPC codes where the puncturing patterns are either random or possess some structure.

The paper was published in the IEEE Trans. on Information Theory, vol. 53, no. 2, pp. 580 - 598, February 2007. It was submitted in August 17, 2005, and revised in October 11, 2006. The material in this paper was presented in part at the 2006 IEEE International Symposium on Information Theory (ISIT 2006), Seattle, Washington, USA, July 2006. Communicated by Tom Richardson, Associate Editor for Coding Theory.

The authors are with the Department of Electrical Engineering, Technion-Israel Institute of Technology, Haifa 32000, Israel. E-mails: \{sason@ee, igillw@tx\}.technion.ac.il. 
The performance of punctured LDPC codes under ML decoding was studied in [2] via analyzing the asymptotic growth rate of their average weight distributions and using upper bounds on the decoding error probability under ML decoding. Based on this analysis, it was proved that for any MBIOS channel, capacity-achieving codes of any desired rate can be constructed by puncturing the code bits of ensembles of LDPC codes whose design rate (before puncturing) is sufficiently low. The performance of punctured LDPC codes over the AWGN channel was studied in [4] under message-passing iterative (MPI) decoding. Ha and McLaughlin studied in [4] two methods for puncturing LDPC codes where the first method assumes random puncturing of the code bits at a fixed rate, and the second method assumes possibly different puncturing rates for each subset of code bits which corresponds to variable nodes of a fixed degree. For the second approach, called 'intentional puncturing', the degree distributions of the puncturing patterns were optimized in [4], [5] where, for a given design rate, it was aimed to minimize the gap to capacity under iterative decoding (by using the Gaussian approximation); exact values of these optimized puncturing patterns were also calculated by the density evolution analysis and show good agreement with the Gaussian approximation. The results in [4], [5] exemplify the usefulness of punctured LDPC codes for a relatively wide range of rates, and therefore, they are suitable for rate-compatible puncturing.

The transmission of punctured codes over a single channel can be regarded as a special case of communication of the original code over a set of parallel channels (where this set of channels is defined by the different puncturing rates applied to disjoint subsets of the code bits). We therefore apply the bounds derived in this paper to the special case of the transmission of ensembles of punctured LDPC codes over an arbitrary MBIOS channel. Theorems related to the achievable rates and decoding complexity of punctured LDPC codes are derived. For ensembles of punctured LDPC codes, the calculation of bounds on their thresholds under ML decoding and their exact thresholds under iterative decoding (based on the density evolution analysis) is of interest; it enables one to distinguish between the asymptotic loss in performance which follows from the sub-optimality of the iterative decoder and the inherent loss in performance which is attributed to the structure of the codes (so it therefore exists even under ML decoding).

The paper is organized as follows: Section II derives bounds on the conditional entropy of the transmitted codeword given the received sequence at the output of the parallel channels where the component channels are considered to be MBIOS. Section III relies on the previous bounds and derives an upper bound on the achievable rates of LDPC codes under ML decoding where these codes are transmitted over parallel MBIOS channels. Section IV uses the latter result for the derivation of upper bounds on the achievable rates of ensembles of randomly and intentionally punctured LDPC codes whose transmission takes place over an arbitrary MBIOS channel, and numerical results are exemplified for various ensembles. Section V provides a lower bound on the decoding complexity (per iteration) of ensembles of LDPC codes under MPI decoding for parallel MBIOS channels. The latter result is used for the derivation of lower bounds on the decoding complexity of randomly and intentionally punctured LDPC codes for MBIOS channels; looser versions of these bounds suggest a simplified re-derivation of previously reported bounds on the decoding complexity of randomly punctured LDPC codes (as shown in the appendix). Finally, Section VI summarizes our discussion, and presents a diagram which shows interconnections between the theorems introduced in this paper and some other previously reported results from [1], [9], [10], [12], [14], [17]. The preliminary material on ensembles of LDPC codes and notation required for this paper are introduced in [13] and [17, Section 2].

\section{Bounds on the Conditional Entropy for PARAllel Channels}

This section serves as a preparatory step towards the derivation of upper bounds on the achievable rates of ML decoded binary linear block codes whose transmission takes place over statistically independent parallel MBIOS channels. To this end, we present in this section upper and lower bounds on the conditional entropy of the transmitted codeword given the received sequence at the output of these channels.

\section{A. Lower Bound on the Conditional Entropy}

We begin by deriving an information-theoretic lower bound on the conditional entropy of the transmitted codeword given the received sequence, when the transmission takes place over a set of $J$ independent parallel MBIOS channels.

Proposition 2.1: Let $\mathcal{C}$ be a binary linear block code of length $n$, and assume that its transmission takes place over a set of $J$ statistically independent parallel MBIOS channels. Let $C_{j}$ denote the capacity of the $j^{\text {th }}$ channel (in bits per channel use), and $a(\cdot ; j)$ designate the conditional pdf of the log-likelihood ratio (LLR) at the output of the 
$j^{\text {th }}$ channel given its input is 0 . Let $\mathbf{X}=\left(X_{1}, \ldots X_{n}\right)$ and $\mathbf{Y}=\left(Y_{1}, \ldots, Y_{n}\right)$ designate the transmitted codeword and received sequence, respectively, $\mathcal{I}(j)$ be the set of indices of the code bits transmitted over the $j^{\text {th }}$ channel, $n^{[j]} \triangleq|\mathcal{I}(j)|$ be the size of this set, and $p_{j} \triangleq \frac{n^{[j]}}{n}$ be the fraction of bits transmitted over the $j^{\text {th }}$ channel. For an arbitrary $c \times n$ parity-check matrix $H$ of the code $\mathcal{C}$, let $\beta_{j, m}$ designate the number of indices in $\mathcal{I}(j)$ referring to bits which are involved in the $m^{\text {th }}$ parity-check equation of $H$ (where $m \in\{1, \ldots, c\}$ ), and let $R_{\mathrm{d}}=1-\frac{c}{n}$ be the design rate of $\mathcal{C}$. Then, the conditional entropy of the transmitted codeword given the received sequence satisfies

$$
\begin{aligned}
\frac{H(\mathbf{X} \mid \mathbf{Y})}{n} \geq 1 & -\sum_{j=1}^{J} p_{j} C_{j}-\left(1-R_{\mathrm{d}}\right) \\
& \cdot\left(1-\frac{1}{2 n\left(1-R_{\mathrm{d}}\right) \ln 2}\right. \\
& \left.\cdot \sum_{p=1}^{\infty}\left\{\frac{1}{p(2 p-1)} \sum_{m=1}^{n\left(1-R_{\mathrm{d}}\right)} \prod_{j=1}^{J}\left(g_{j, p}\right)^{\beta_{j, m}}\right\}\right)
\end{aligned}
$$

where

$$
g_{j, p} \triangleq \int_{0}^{\infty} a(l ; j)\left(1+e^{-l}\right) \tanh ^{2 p}\left(\frac{l}{2}\right) \mathrm{d} l, \quad \begin{aligned}
& j \in\{1, \ldots, J\} . \\
& p \in \mathbb{N}
\end{aligned} .
$$

Remark 2.1: Note that the input vector $\mathbf{X}$ is chosen uniformly at random from the codewords of a binary linear block code. Each input bit $X_{i}$ therefore either gets the values 0 or 1 with probability $\frac{1}{2}$ or is set to zero (due to the linearity of the code). In the following proof, we assume that all the code symbols get the values 0 or 1 with equal probability. By slightly modifying the proof, it is simple to show that the bound also holds for the other case where some of the code bits are set to zero.

Proof: The proof relies on concepts which are presented in [1], [17], and generalizes them to the case of parallel channels. If a symbol is transmitted over the $j^{\text {th }}$ MBIOS channel and $y$ is the corresponding output, then the LLR gets the form

$$
\operatorname{LLR}(y ; j)=\ln \left(\frac{p(y \mid 0 ; j)}{p(y \mid 1 ; j)}\right), \quad j \in\{1, \ldots, J\}, \quad y \in \mathcal{Y}
$$

where $\mathcal{Y}$ denotes the output alphabet of each component channel, ${ }^{1}$ and $p(\cdot \mid \cdot ; j)$ is the conditional pdf of the $j^{\text {th }}$ channel. For each one of these $J$ component channels, we move from the original mapping of $X \rightarrow Y$ (where according to (1), each symbol is transmitted over only one of these $J$ channels) to an equivalent representation of the channel $X \rightarrow \widetilde{Y}$, where $\widetilde{Y}$ represents the LLR of the channel output $Y$. These channels are equivalent in the sense that $H(X \mid \widetilde{Y})=H(X \mid Y)$. The basic idea for showing the equivalence between the original set of parallel channels and the one which will be introduced shortly is based on the principle that the LLR forms a sufficient statistics of an MBIOS channel.

In the following, we characterize an equivalent channel to each of the $J$ parallel channels. The output of the equivalent channel is defined to be $\widetilde{Y} \triangleq(\Phi, \Omega)$. For the $j$-th channel, $\widetilde{Y}$ is calculated from $Y$ as follows:

$$
\begin{aligned}
& \Omega \triangleq|\operatorname{LLR}(Y ; j)| \\
& \Phi \triangleq\left\{\begin{array}{ll}
0 & \text { if } \operatorname{LLR}(Y ; j)>0 \\
1 & \text { if } \operatorname{LLR}(Y ; j)<0 \\
0 \text { or } 1 \text { w.p. } \frac{1}{2} & \text { if } \operatorname{LLR}(Y ; j)=0
\end{array} .\right.
\end{aligned}
$$

Due to the symmetry of the communication channel, the equivalent channel can be seen as a channel with additive noise where the transmitted signal affects only the $\Phi$ component of the output $\widetilde{Y}$. The characterization of the equivalent channel in this form is used for the continuation of this proof and is presented below. For each index $i \in \mathcal{I}(j)$, let us choose independently a value $L_{i}$ according to the conditional pdf $a(\cdot ; j)$, given the input symbol

\footnotetext{
${ }^{1}$ In case the output alphabets of the component channels are not equal, then $\mathcal{Y}$ can be defined as their union.
} 
is zero. For $i \in\{1, \ldots, n\}$, let

$$
\Omega_{i} \triangleq\left|L_{i}\right|, \quad \Theta_{i} \triangleq\left\{\begin{array}{lll}
0 & \text { if } & L_{i}>0 \\
1 & \text { if } & L_{i}<0 \\
0 \text { or } 1 \text { w.p. } \frac{1}{2} & \text { if } & L_{i}=0
\end{array} .\right.
$$

The output of the set of equivalent channels is defined to be $\widetilde{\mathbf{Y}}=\left(\widetilde{Y}_{1}, \ldots, \widetilde{Y}_{n}\right)$ where $\widetilde{Y}_{i}=\left(\Phi_{i}, \Omega_{i}\right)$ and $\Phi_{i}=\Theta_{i}+X_{i}$ where the addition is modulo 2. This defines the mapping

$$
X \rightarrow \tilde{Y}=(\Phi, \Omega)
$$

where $\Phi$ is a binary random variable which is affected by $X$, and $\Omega$ is a non-negative random variable which is independent of $X$. Note that due to the symmetry of the parallel channels, for each index $i \in \mathcal{I}(j)$, the joint distribution of $\left(\Phi_{i}, \Omega_{i}\right)$ is independent of $i$, and is equal to the distribution of the pair representing the sign and magnitude of $\operatorname{LLR}(Y ; j)$. Hence,

$$
f_{\Omega_{i}}(\omega) \triangleq f_{\Omega}(\omega ; j)= \begin{cases}a(\omega ; j)+a(-\omega ; j) & \text { if } \omega>0 \\ =\left(1+e^{-\omega}\right) a(\omega ; j) & \text { if } \omega=0 \\ a(0 ; j) & \text { if } \quad \omega\end{cases}
$$

where we rely on the symmetry property of $a(\cdot ; j)$.

Denoting by $R$ the rate of the code $\mathcal{C}$, since the codewords are transmitted with equal probability

$$
H(\mathbf{X})=n R \text {. }
$$

Also, since the $J$ parallel channels are memoryless, then

$$
H(\mathbf{Y} \mid \mathbf{X})=\sum_{i=1}^{n} H\left(Y_{i} \mid X_{i}\right)
$$

The mapping $Y_{i} \rightarrow \tilde{Y}_{i}$ is memoryless, hence

$$
H(\tilde{\mathbf{Y}} \mid \mathbf{Y})=\sum_{i=1}^{n} H\left(\widetilde{Y}_{i} \mid Y_{i}\right)
$$

and

$$
\begin{aligned}
H(\mathbf{Y}) & =H(\widetilde{\mathbf{Y}})-H(\tilde{\mathbf{Y}} \mid \mathbf{Y})+H(\mathbf{Y} \mid \widetilde{\mathbf{Y}}) \\
& =H(\tilde{\mathbf{Y}})-\sum_{i=1}^{n} H\left(\tilde{Y}_{i} \mid Y_{i}\right)+H(\mathbf{Y} \mid \widetilde{\mathbf{Y}}) \\
H(\mathbf{Y} \mid \tilde{\mathbf{Y}}) & \leq \sum_{i=1}^{n} H\left(Y_{i} \mid \widetilde{Y}_{i}\right) \\
& =\sum_{i=1}^{n}\left[H\left(Y_{i}\right)-H\left(\tilde{Y}_{i}\right)+H\left(\tilde{Y}_{i} \mid Y_{i}\right)\right] .
\end{aligned}
$$


Applying the above towards the derivation of a lower bound on the conditional entropy $H(\mathbf{X} \mid \mathbf{Y})$, we get

$$
\begin{aligned}
& H(\mathbf{X} \mid \mathbf{Y})=H(\mathbf{X})+H(\mathbf{Y} \mid \mathbf{X})-H(\mathbf{Y}) \\
& \stackrel{(a)}{=} n R+\sum_{i=1}^{n} H\left(Y_{i} \mid X_{i}\right)-H(\widetilde{\mathbf{Y}})-H(\mathbf{Y} \mid \widetilde{\mathbf{Y}}) \\
& +\sum_{i=1}^{n} H\left(\widetilde{Y}_{i} \mid Y_{i}\right) \\
& \stackrel{(b)}{\geq} n R+\sum_{i=1}^{n} H\left(Y_{i} \mid X_{i}\right)-H(\widetilde{\mathbf{Y}})+\sum_{i=1}^{n} H\left(\tilde{Y}_{i} \mid Y_{i}\right) \\
& -\sum_{i=1}^{n}\left[H\left(Y_{i}\right)-H\left(\tilde{Y}_{i}\right)+H\left(\widetilde{Y}_{i} \mid Y_{i}\right)\right] \\
& =n R-H(\widetilde{\mathbf{Y}})+\sum_{i=1}^{n} H\left(\widetilde{Y}_{i}\right) \\
& -\sum_{i=1}^{n}\left[H\left(Y_{i}\right)-H\left(Y_{i} \mid X_{i}\right)\right] \\
& =n R-H(\widetilde{\mathbf{Y}})+\sum_{i=1}^{n} H\left(\widetilde{Y}_{i}\right)-\sum_{i=1}^{n} I\left(X_{i} ; Y_{i}\right) \\
& \stackrel{(c)}{\geq} n R-H(\widetilde{\mathbf{Y}})+\sum_{i=1}^{n} H\left(\widetilde{Y}_{i}\right)-\sum_{j=1}^{J} n^{[j]} C_{j}
\end{aligned}
$$

where (a) relies on (5)-(7), (b) relies on (8), and (c) follows since $I\left(X_{i} ; Y_{i}\right) \leq C_{j}$ for all $i \in \mathcal{I}(j)$, and $|\mathcal{I}(j)|=n^{[j]}$ for $j \in\{1, \ldots, J\}$. In order to obtain a lower bound on $H(\mathbf{X} \mid \mathbf{Y})$ from (9), we calculate the entropy of the random variables $\left\{\widetilde{Y}_{i}\right\}$, and find an upper bound on the entropy of the random vector $\widetilde{\mathbf{Y}}$. This finally provides the lower bound on the conditional entropy given in (2). Considering an index $i \in \mathcal{I}(j)$ for some $j \in\{1,2, \ldots J\}$, we get

$$
\begin{aligned}
H\left(\widetilde{Y}_{i}\right) & =H\left(\Phi_{i}, \Omega_{i}\right) \\
& =H\left(\Omega_{i}\right)+H\left(\Phi_{i} \mid \Omega_{i}\right) \\
& =H\left(\Omega_{i}\right)+E_{\omega}\left[H\left(\Phi_{i} \mid \Omega_{i}=\omega\right)\right] \\
& =H\left(\Omega_{i}\right)+1
\end{aligned}
$$

where the last transition is due to the fact that given the absolute value of the LLR, since the parallel channels are MBIOS and the coded bits are equally likely to be 0 or 1 (see Remark 2.1), the sign of the LLR is equally likely to be positive or negative. The entropy $H\left(\Omega_{i}\right)$ is not expressed explicitly as it cancels out later.

We now turn to derive an upper bound on $H(\widetilde{\mathbf{Y}})$ :

$$
\begin{aligned}
H(\widetilde{\mathbf{Y}}) & =H\left(\left(\Phi_{1}, \ldots, \Phi_{n}\right),\left(\Omega_{1}, \ldots, \Omega_{n}\right)\right) \\
& =H\left(\Omega_{1}, \ldots, \Omega_{n}\right)+H\left(\left(\Phi_{1}, \ldots, \Phi_{n}\right) \mid\left(\Omega_{1}, \ldots, \Omega_{n}\right)\right) \\
& =\sum_{i=1}^{n} H\left(\Omega_{i}\right)+H\left(\left(\Phi_{1}, \ldots, \Phi_{n}\right) \mid\left(\Omega_{1}, \ldots, \Omega_{n}\right)\right)
\end{aligned}
$$

where the last equality follows since the random variables $\Omega_{i}$ are statistically independent.

Define the $c$-dimensional syndrome vector as

$$
\mathbf{S} \triangleq\left(\Phi_{1}, \ldots, \Phi_{n}\right) H^{T}
$$

where $H$ is a $c \times n$ parity-check matrix of the binary linear block code $\mathcal{C}$, and let $M$ be the index of the vector $\left(\Phi_{1}, \ldots, \Phi_{n}\right)$ in the coset which corresponds to $\mathbf{S}$. Since each coset contains $2^{n R}$ elements which are equally likely 
then $H(M)=n R$, and we get

$$
\begin{aligned}
& H\left(\left(\Phi_{1}, \ldots, \Phi_{n}\right) \mid\left(\Omega_{1}, \ldots, \Omega_{n}\right)\right) \\
& =H\left(\mathbf{S}, M \mid\left(\Omega_{1}, \ldots, \Omega_{n}\right)\right) \\
& \leq H(M)+H\left(\mathbf{S} \mid\left(\Omega_{1}, \ldots, \Omega_{n}\right)\right) \\
& =n R+H\left(\mathbf{S} \mid\left(\Omega_{1}, \ldots, \Omega_{n}\right)\right) \\
& \leq n R+\sum_{m=1}^{c} H\left(S_{m} \mid\left(\Omega_{1}, \ldots, \Omega_{n}\right)\right) .
\end{aligned}
$$

Since $\mathbf{X} H^{T}=0$ for any codeword $\mathbf{X} \in \mathcal{C}$, and $\Phi_{i}=\Theta_{i}+X_{i}$ for all $i \in\{1, \ldots, n\}$, then $\mathbf{S}=\left(\Theta_{1}, \ldots, \Theta_{n}\right) H^{T}$.

Let us consider the $m^{\text {th }}$ parity-check equation which involves $k_{m}$ variables, and assume that the set of indices of these variables is $\left\{i_{1}, \ldots, i_{k_{m}}\right\}$. Then, the component $S_{m}$ of the syndrome is equal to 1 if and only if there is an odd number of ones in the random vector $\left(\Theta_{i_{1}}, \ldots, \Theta_{i_{k_{m}}}\right)$. To calculate the probability that $S_{m}$ is equal to 1 , we rely on the following lemma:

Lemma 2.1 ([17], Lemma 4.1): If the $m^{\text {th }}$ linear constraint defined by the parity-check matrix $H$ involves $k_{m}$ variables, and if $\left\{i_{1}, \ldots, i_{k_{m}}\right\}$ denote the indices of these variables, then

From this lemma, we obtain

$$
\begin{aligned}
& \operatorname{Pr}\left(S_{m}=1 \mid\left(\Omega_{i_{1}}, \ldots, \Omega_{i_{k_{m}}}\right)=\left(\alpha_{1}, \ldots, \alpha_{k_{m}}\right)\right) \\
& =\frac{1}{2}\left[1-\prod_{w=1}^{k_{m}} \tanh \left(\frac{\alpha_{w}}{2}\right)\right] .
\end{aligned}
$$

$$
\begin{aligned}
& H\left(S_{m} \mid\left(\Omega_{i_{1}}, \ldots, \Omega_{i_{k_{m}}}\right)=\left(\alpha_{1}, \ldots, \alpha_{k_{m}}\right)\right) \\
& =h_{2}\left(\frac{1}{2}\left[1-\prod_{w=1}^{k_{m}} \tanh \left(\frac{\alpha_{w}}{2}\right)\right]\right)
\end{aligned}
$$

where $h_{2}$ denotes the binary entropy function to base 2. By taking the statistical expectation over the $k_{m}$ random variables $\Omega_{i_{1}}, \ldots, \Omega_{i_{k_{m}}}$, we get

$$
\begin{array}{r}
H\left(S_{m} \mid\left(\Omega_{i_{1}}, \ldots, \Omega_{i_{k_{m}}}\right)\right) \\
=\int_{0}^{\infty} \ldots \int_{0}^{\infty} h_{2}\left(\frac{1}{2}\left[1-\prod_{w=1}^{k_{m}} \tanh \left(\frac{\alpha_{w}}{2}\right)\right]\right) \\
\cdot \prod_{w=1}^{k_{m}} f_{\Omega_{i_{w}}}\left(\alpha_{w}\right) d \alpha_{1} d \alpha_{2} \ldots d \alpha_{k_{m}} .
\end{array}
$$

Let $\beta_{j, m}$ denote the number of indices $w \in\left\{i_{1}, \ldots, i_{k_{m}}\right\}$ referring to variables which are transmitted over the $j^{\text {th }}$ channel. From the Taylor series expansion of the binary entropy function $\left(h_{2}\right)$ around $x=\frac{1}{2}$ (see [17, Appendix B.1])

$$
h_{2}(x)=1-\frac{1}{2 \ln 2} \sum_{p=1}^{\infty} \frac{(1-2 x)^{2 p}}{p(2 p-1)}, \quad 0 \leq x \leq 1
$$

it follows that

$$
\begin{aligned}
& H\left(S_{m} \mid\left(\Omega_{i_{1}}, \ldots, \Omega_{i_{k_{m}}}\right)\right) \\
& =1-\frac{1}{2 \ln 2} \sum_{p=1}^{\infty}\left\{\frac{1}{p(2 p-1)}\right. \\
& \left.\cdot \prod_{w=1}^{k_{m}}\left(\int_{0}^{\infty} f_{\Omega_{i_{w}}}(\alpha) \tanh ^{2 p}\left(\frac{\alpha}{2}\right) d \alpha\right)\right\} \\
& =1-\frac{1}{2 \ln 2} \sum_{p=1}^{\infty}\left\{\frac{1}{p(2 p-1)}\right.
\end{aligned}
$$




$$
\left.\cdot \prod_{j=1}^{J}\left(\int_{0}^{\infty} f_{\Omega}(\alpha ; j) \tanh ^{2 p}\left(\frac{\alpha}{2}\right) d \alpha\right)^{\beta_{j, m}}\right\}
$$

where the first transition is based on (14) and follows along the same lines as [17, Appendix B.2]), and the second transition is due to the fact that for all $i \in \mathcal{I}(j)$, the pdf of the random variable $\Omega_{i}$ is independent of $i$, see (4). Summing over all the parity-check equations of $H$ gives

$$
\begin{aligned}
& \sum_{m=1}^{c} H\left(S_{m} \mid\left(\Omega_{1}, \ldots, \Omega_{n}\right)\right) \\
&=c- \frac{1}{2 \ln 2} \sum_{p=1}^{\infty}\left\{\frac{1}{p(2 p-1)}\right. \\
&\left.\cdot \sum_{m=1}^{c}\left[\prod_{j=1}^{J}\left(\int_{0}^{\infty} f_{\Omega}(\alpha ; j) \tanh ^{2 p}\left(\frac{\alpha}{2}\right) d \alpha\right)^{\beta_{j, m}}\right]\right\} .
\end{aligned}
$$

By combining (4), (11), (12) and (16), we get the following upper bound on $H(\widetilde{\mathbf{Y}})$ :

$$
\begin{aligned}
H(\widetilde{\mathbf{Y}}) \leq & \sum_{i=1}^{n} H\left(\Omega_{i}\right)+n R \\
& +c\left[1-\frac{1}{2 c \ln 2} \sum_{p=1}^{\infty} \frac{1}{p(2 p-1)} \sum_{m=1}^{c}\left\{\prod _ { j = 1 } ^ { J } \left(\int_{0}^{\infty} a(\alpha ; j)\right.\right.\right. \\
\left.\left.\left.\cdot\left(1+e^{-\alpha}\right) \tanh ^{2 p}\left(\frac{\alpha}{2}\right) d \alpha\right)^{\beta_{j, m}}\right\}\right] & \stackrel{(a)}{=} \sum_{i=1}^{n} H\left(\Omega_{i}\right)+n R+n\left(1-R_{\mathrm{d}}\right)\left[1-\frac{1}{2 n\left(1-R_{\mathrm{d}}\right) \ln 2}\right. \\
& \left.\cdot \sum_{p=1}^{\infty}\left\{\frac{1}{p(2 p-1)} \sum_{m=1}^{c} \prod_{j=1}^{J} g_{j, p}^{\beta_{j, m}}\right\}\right]
\end{aligned}
$$

where $(a)$ follows from the definition of $g_{j, p}$ in (3), and since $R_{\mathrm{d}} \triangleq 1-\frac{c}{n}$ denotes the design rate of $\mathcal{C}$. Finally, the substitution of (10) and (17) in the RHS of (9) provides the lower bound on the conditional entropy $H(\mathbf{X} \mid \mathbf{Y})$ given in (2). This completes the proof of the proposition.

\section{B. Upper Bound on the Conditional Entropy}

In this section, we provide an upper bound on the conditional entropy of the transmitted codeword given the received sequence. The bound holds for an arbitrary binary linear block code whose transmission takes place over a set of parallel channels, and is expressed in terms of the code rate and the bit-error probability of the code (under ML decoding or a sub-optimal decoding algorithm).

Lemma 2.2: Let $\mathcal{C}$ be a binary linear block code of length $n$ and rate $R$, and assume that its transmission takes place over a set of parallel channels. Let $\mathbf{X}=\left(X_{1}, \ldots, X_{n}\right)$ and $\mathbf{Y}=\left(Y_{1}, \ldots, Y_{n}\right)$ designate the transmitted codeword and the received sequence, respectively. Then

$$
\frac{H(\mathbf{X} \mid \mathbf{Y})}{n} \leq R h_{2}\left(P_{\mathrm{b}}\right)
$$

where $P_{\mathrm{b}}$ designates the bit error probability of the code $\mathcal{C}$ under an arbitrary decoding algorithm.

Proof: See the proof in [17, Appendix I-A], which holds regardless of the model of the communication channel. 


\section{An UpPer bound on the Achievable Rates of LDPC CODES OVER PARAllel Channels}

In this section, we derive an upper bound on the design rate of a sequence of ensembles of LDPC codes whose transmission takes place over a set of statistically independent parallel MBIOS channels, and which achieves vanishing bit error probability under ML decoding. This bound is used in the next section for the derivation of an upper bound on the design rate of an arbitrary sequence of ensembles of punctured LDPC codes.

Let us assume that a binary LDPC code $\mathcal{C}$ of length $n$ is transmitted over a set of $J$ statistically independent parallel MBIOS channels. Denote the number of code bits of $\mathcal{C}$ which are transmitted over the $j^{\text {th }}$ channel by $n^{[j]}$, and the fraction of bits transmitted over the $j^{\text {th }}$ channel by

$$
p_{j} \triangleq \frac{n^{[j]}}{n}, \quad j \in\{1, \ldots, J\} .
$$

Let $\mathcal{G}$ be a bipartite graph which represents the $\operatorname{code} \mathcal{C}$, and $E$ be the set of edges in $\mathcal{G}$. Let $E^{[j]}$ designate the set of edges connected to variable nodes which correspond to code bits transmitted over the $j^{\text {th }}$ channel, and

$$
q_{j} \triangleq \frac{\left|E^{[j]}\right|}{|E|}, \quad j \in\{1, \ldots, J\}
$$

denote the fraction of edges connected to these variable nodes. Referring to the edges from the subset $E^{[j]}$, let $\lambda_{i}^{[j]}$ designate the fraction of these edges which are connected to variable nodes of degree $i$, and define the following $J$ degree distributions from the edge perspective:

$$
\lambda^{[j]}(x) \triangleq \sum_{i=2}^{\infty} \lambda_{i}^{[j]} x^{i-1}, \quad j \in\{1, \ldots, J\}
$$

which correspond to each of the $J$ parallel channels. According to this notation, the number of edges connected to variable nodes corresponding to code bits transmitted over the $j^{\text {th }}$ channel is given by

$$
\left|E^{[j]}\right|=\frac{n^{[j]}}{\sum_{i=2}^{\infty} \frac{\lambda_{i}^{[j]}}{i}}, \quad j \in\{1, \ldots, J\} .
$$

For the simplicity of the notation, let us define a vector of degree distributions for the variable nodes from the edge perspective to be $\underline{\lambda}(x)=\left(\lambda^{[1]}(x), \ldots, \lambda^{[J]}(x)\right)$. Following the notation in [10], the ensemble $(n, \underline{\lambda}, \rho)$ is defined to be the set of LDPC codes of length $n$, which according to their representation by bipartite graphs and the assignment of their code bits to the parallel channels, imply left and right degree distributions of $\underline{\lambda}$ and $\rho$, respectively.

Lemma 3.1:

$$
\frac{1}{\int_{0}^{1} \lambda(x) \mathrm{d} x}=\sum_{j=1}^{J}\left\{\frac{p_{j}}{\int_{0}^{1} \lambda^{[j]}(x) \mathrm{d} x}\right\}
$$

where $\lambda$ is the overall left degree distribution which serves to construct the vector of left degree distributions $\underline{\lambda}$ by considering the assignments of variables nodes to the $J$ parallel channels.

Proof: Since $E^{[1]}, \ldots, E^{[J]}$ form a sequence of disjoint sets whose union is the set $E$, we get $|E|=\sum_{j=1}^{J}\left|E^{[j]}\right|$. From (21), we therefore get

$$
\frac{n}{\sum_{i=2}^{\infty} \frac{\lambda_{i}}{i}}=\sum_{j=1}^{J}\left\{\frac{n^{[j]}}{\sum_{i=2}^{\infty} \frac{\lambda_{i}^{[j]}}{i}}\right\}
$$

By dividing both sides of this equality by $n$, and using (19) and the equality $\sum_{i} \frac{\lambda_{i}}{i}=\int_{0}^{1} \lambda(x) \mathrm{d} x$, the lemma follows immediately.

Lemma 3.2:

$$
q_{j}=\frac{p_{j}}{\int_{0}^{1} \lambda^{[j]}(x) \mathrm{d} x} \cdot \frac{1}{\sum_{k=1}^{J}\left\{\frac{p_{k}}{\int_{0}^{1} \lambda^{[k]}(x) \mathrm{d} x}\right\}}, \quad \forall j \in\{1, \ldots, J\} .
$$


Proof: The lemma follows directly from (20), (21) and Lemma 3.1.

In the following, we introduce a sequence of ensembles of binary LDPC codes, $\left\{\left(n_{r}, \underline{\lambda}_{r}, \rho\right)\right\}_{r=1}^{\infty}$, where all the codes in each ensemble have the same number of bits assigned to each of the $J$ parallel channels. The right degree distribution $\rho$ is assumed to be fixed for all the ensembles of this sequence (i.e., it is independent of $r$ ), and it is also assumed to have a bounded maximal degree (which corresponds to a bounded maximal degree of the parity-check nodes). We assume that $\lambda$, which corresponds to the overall left degree distribution of the edges, is also independent of $r$; due of the independence of $\lambda$ and $\rho$ in $r$, one can consider here the common design rate of the sequence of ensembles $\left\{\left(n_{r}, \underline{\lambda}_{r}, \rho\right)\right\}_{r=1}^{\infty}$ which does not depend on $r$.

This setting is general enough for applying the following theorem to various applications which form particular cases of communication over parallel channels, e.g., punctured LDPC codes [2], [4], non-uniformly error protected LDPC codes [10], and LDPC-coded modulation (see e.g., [6], [16]). In this setting, the fraction of code bits assigned to the $j^{\text {th }}$ channel, $p_{j, r}$, depends on $j \in\{1, \ldots, J\}$ and $r \in \mathbb{N}$, but not on the particular code chosen from each ensemble. It follows from Lemma 3.2 that the same property also holds for $q_{j, r}$ which designates the fraction of edges connected to variable nodes whose code bits are assigned to the $j^{\text {th }}$ channel. In the following, we assume that the limits

$$
p_{j} \triangleq \lim _{r \rightarrow \infty} p_{j, r}, \quad q_{j} \triangleq \lim _{r \rightarrow \infty} q_{j, r}
$$

exist and also that they are positive for all $j \in\{1, \ldots, J\}$ (though in general, they are non-negative).

Theorem 3.1: Let a sequence of ensembles of binary LDPC codes, $\left\{\left(n_{r}, \underline{\lambda}_{r}, \rho\right)\right\}_{r=1}^{\infty}$, be transmitted over a set of $J$ statistically independent parallel MBIOS channels, and assume that the block length $\left(n_{r}\right)$ goes to infinity as we let $r$ tend to infinity. Let $C_{j}$ denote the capacity of the $j^{\text {th }}$ channel, and $a(\cdot ; j)$ designate the pdf of the LLR at the output of the $j^{\text {th }}$ channel, given its input symbol is zero. If in the limit where $r$ tends to infinity, the bit error probability of this sequence vanishes under ML decoding, then the common design rate $R_{\mathrm{d}}$ of these ensembles satisfies

$$
R_{\mathrm{d}} \leq 1-\frac{1-\sum_{j=1}^{J} p_{j} C_{j}}{1-\frac{1}{2 \ln 2} \sum_{p=1}^{\infty}\left\{\frac{1}{p(2 p-1)} \Gamma\left(\sum_{j=1}^{J} q_{j} g_{j, p}\right)\right\}}
$$

where $\Gamma$ denotes the right degree distribution from the node perspective, and $g_{j, p}$ is introduced in (3).

Proof: Let $\left\{\mathcal{C}_{r}\right\}_{r=1}^{\infty}$ be a sequence of binary LDPC codes chosen uniformly at random from the sequence of ensembles $\left\{\left(n_{r}, \underline{\lambda}_{r}, \rho\right)\right\}_{r=1}^{\infty}$. Denote the rate of the code $\mathcal{C}_{r}$ by $R_{r}$, and let $P_{\mathrm{b}, r}$ be its bit error probability under ML decoding. Let $\mathcal{G}_{r}$ be a bipartite graph of the code $\mathcal{C}_{r}$ whose left and right degree distributions from the edge perspective are $\underline{\lambda}_{r}$ and $\rho$, respectively. From Proposition 2.1 and Lemma 2.2, it follows that the following inequality holds for the binary linear block code $\mathcal{C}_{r}$ :

$$
\begin{aligned}
& R_{r} h_{2}\left(P_{\mathrm{b}, r}\right) \\
& \geq 1-\sum_{j=1}^{J} p_{j, r} C_{j} \\
& -\left(1-R_{\mathrm{d}}\right)\left(1-\frac{1}{2 n_{r}\left(1-R_{\mathrm{d}}\right) \ln 2}\right. \\
& \left.\cdot \sum_{p=1}^{\infty}\left\{\frac{1}{p(2 p-1)} \sum_{m=1}^{n_{r}\left(1-R_{\mathrm{d}}\right)} \prod_{j=1}^{J}\left(g_{j, p}\right)^{\beta_{r, j, m}}\right\}\right)
\end{aligned}
$$

where $n_{r}$ is the block length of the code $\mathcal{C}_{r}, R_{\mathrm{d}}$ is the common design rate for all the codes from the sequence of ensembles $\left\{\left(n_{r}, \underline{\lambda}_{r}, \rho\right)\right\}_{r=1}^{\infty}$, and $\beta_{r, j, m}$ denotes the number of edges which are connected to the $m^{\text {th }}$ parity-check node of the graph $\mathcal{G}_{r}$ and are related to code bits transmitted over the $j^{\text {th }}$ channel (where $j \in\{1, \ldots, J\}$ and 
$\left.m \in\left\{1, \ldots n_{r}\left(1-R_{\mathrm{d}}\right)\right\}\right)$. By taking the expectation on both sides of (27) and letting $r$ tend to infinity, we get

$$
\begin{aligned}
0 \geq 1 & -\sum_{j=1}^{J} p_{j} C_{j}-\left(1-R_{\mathrm{d}}\right) \\
\cdot \lim _{r \rightarrow \infty}\left(1-\frac{1}{2 n_{r}\left(1-R_{\mathrm{d}}\right) \ln 2}\right. & \left.\cdot \sum_{p=1}^{\infty}\left\{\frac{1}{p(2 p-1)} \sum_{m=1}^{n_{r}\left(1-R_{\mathrm{d}}\right)} \mathbb{E}\left(\prod_{j=1}^{J}\left(g_{j, p}\right)^{\beta_{r, j, m}}\right)\right\}\right) .
\end{aligned}
$$

The LHS of (28) follows from the LHS of (27), due to the concavity of the binary entropy function and Jensen's inequality, and since by our assumption, the bit error probability of the ensembles vanishes in the limit where $r$ tends to infinity.

The derivation of an upper bound on the design rate is proceeded by calculating the expectation of the product inside the RHS of (28). Let $k_{r, m}$ denote the degree of the $m^{\text {th }}$ parity-check node of the bipartite graph $\mathcal{G}_{r}$, then the smoothing theorem gives

$$
\begin{aligned}
& \mathbb{E}\left(\prod_{j=1}^{J}\left(g_{j, p}\right)^{\beta_{r, j, m}}\right) \\
& =\mathbb{E}\left[\mathbb{E}\left(\prod_{j=1}^{J}\left(g_{j, p}\right)^{\beta_{r, j, m}} \mid \sum_{j=1}^{J} \beta_{r, j, m}=k_{r, m}\right)\right]
\end{aligned}
$$

where the outer expectation in the RHS of (29) is carried over the random variable $k_{r, m}$. We begin by calculating the inner expectation in the RHS of (29). It follows from (21) that the number of edges, $\left|E_{r}^{[j]}\right| \triangleq\left|E^{[j]}\left(\mathcal{G}_{r}\right)\right|$, connected to variable nodes corresponding to code bits transmitted over the $j^{\text {th }}$ channel, is independent of the code $\mathcal{C}_{r}$ chosen from the ensemble $\left(n_{r}, \underline{\lambda}_{r}, \rho\right)$. The same property also holds for the total number of edges in the graph (since $\left.\left|E_{r}\right|=\sum_{j=1}^{J}\left|E_{r}^{[j]}\right|\right)$. Since the code $\mathcal{C}_{r}$ is chosen uniformly at random from the ensemble, it follows that if $k_{r, m}$ is a given positive integer, then

$$
\begin{aligned}
& \mathbb{E}\left(\prod_{j=1}^{J}\left(g_{j, p}\right)^{\beta_{r, j, m}} \mid \sum_{j=1}^{J} \beta_{r, j, m}=k_{r, m}\right) \\
& =\sum_{\substack{b_{1}, \ldots, b_{J} \geq 0 \\
\sum_{j=1}^{J} b_{j}=k_{r, m}}}\left\{\operatorname{Pr}\left(\beta_{r, j, m}=b_{j}, \forall j \in\{1, \ldots, J\}\right)\right. \\
& \left.\cdot \prod_{j=1}^{J}\left(g_{j, p}\right)^{b_{j}}\right\} \\
& =\sum_{\substack{b_{1}, \ldots, b_{J} \geq 0 \\
\sum J}}\left\{\frac{\left(\begin{array}{c}
\left|E_{r}^{[1]}\right| \\
b_{1}
\end{array}\right) \cdots\left(\begin{array}{c}
\left|E_{r}^{[J]}\right| \\
b_{J}
\end{array}\right)}{\left(\begin{array}{c}
\left|E_{r}\right| \\
k_{r, m}
\end{array}\right)} \prod_{j=1}^{J}\left(g_{j, p}\right)^{b_{j}}\right\} .
\end{aligned}
$$

Lemma 3.3:

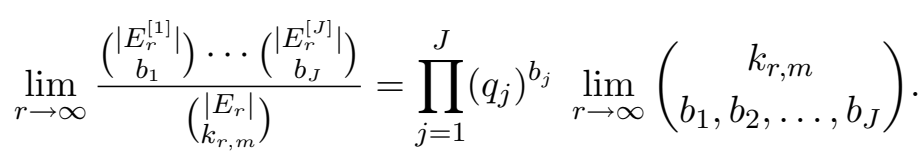

Proof: By assumption, in the limit where we let $r$ tend to infinity, the block length $n_{r}$ also tends to infinity. Hence, from (21) and the assumption that $q_{j}>0$ for every $j \in\{1, \ldots, J\}$, we get that for all $j \in\{1, \ldots, J\}, E_{r}^{[j]}$ 
approaches infinity in the limit where $r$ tends to infinity.

$$
\begin{aligned}
& \lim _{r \rightarrow \infty} \frac{\left(\begin{array}{c}
\left|E_{r}^{[1]}\right| \\
b_{1}
\end{array}\right) \cdots\left(\begin{array}{c}
\left|E_{r}^{[J]}\right| \\
b_{J}
\end{array}\right)}{\left(\begin{array}{c}
\left|E_{r}\right| \\
k_{r, m}
\end{array}\right)} \\
& =\lim _{r \rightarrow \infty}\left\{\frac{\left|E_{r}^{[1]}\right| ! \cdots\left|E_{r}^{[J]}\right| !}{\left|E_{r}\right| !} \frac{\left(\left|E_{r}\right|-k_{r, m}\right) !}{\left(\left|E_{r}^{[1]}\right|-b_{1}\right) ! \cdots\left(\left|E_{r}^{[J]}\right|-b_{J}\right) !}\right. \\
& \left.\cdot\left(\begin{array}{c}
k_{r, m} \\
b_{1}, b_{2}, \ldots, b_{J}
\end{array}\right)\right\} \\
& =\lim _{r \rightarrow \infty}\left\{\frac{\left|E_{r}^{[1]}\right| ! \cdots\left|E_{r}^{[J]}\right| !}{\left|E_{r}\right| !} \frac{\left(\left|E_{r}\right|-k_{r, m}\right) !}{\left(\left|E_{r}^{[1]}\right|-b_{1}\right) ! \ldots\left(\left|E_{r}^{[J]}\right|-b_{J}\right) !}\right\} \\
& \cdot \lim _{r \rightarrow \infty}\left(\begin{array}{c}
k_{r, m} \\
b_{1}, b_{2}, \ldots, b_{J}
\end{array}\right) \\
& \stackrel{(\mathrm{a})}{=} \lim _{r \rightarrow \infty} \frac{\left|E_{r}^{[1]}\right|^{b_{1}} \cdots\left|E_{r}^{[J]}\right|^{b_{J}}}{\left|E_{r}\right|^{k_{r, m}}} \lim _{r \rightarrow \infty}\left(\begin{array}{c}
k_{r, m} \\
b_{1}, b_{2}, \ldots, b_{J}
\end{array}\right) \\
& \stackrel{(\mathrm{b})}{=} \lim _{r \rightarrow \infty}\left(\frac{\left|E_{r}^{[1]}\right|}{\left|E_{r}\right|}\right)^{b_{1}} \cdots\left(\frac{\left|E_{r}^{[J]}\right|}{\left|E_{r}\right|}\right)^{b_{J}} \lim _{r \rightarrow \infty}\left(\begin{array}{c}
k_{r, m} \\
b_{1}, b_{2}, \ldots, b_{J}
\end{array}\right) \\
& \stackrel{\text { (c) }}{=} \lim _{r \rightarrow \infty} \prod_{j=1}^{J}\left(q_{j, r}\right)^{b_{j}} \lim _{r \rightarrow \infty}\left(\begin{array}{c}
k_{r, m} \\
b_{1}, b_{2}, \ldots, b_{J}
\end{array}\right) \\
& =\prod_{j=1}^{J}\left(q_{j}\right)^{b_{j}} \lim _{r \rightarrow \infty}\left(\begin{array}{c}
k_{r, m} \\
b_{1}, b_{2}, \ldots, b_{J}
\end{array}\right)
\end{aligned}
$$

where equality (a) follows since for all $j \in\{1, \ldots, J\},\left|E_{r}^{[j]}\right| \rightarrow \infty$ as we let $r$ tend to infinity, while on the other hand, the maximal right degree (and hence, also $b_{1}, \ldots, b_{J}$ and $k_{r, m}$ ) stay bounded; equality (b) is valid due to the constraint $\sum_{j=1}^{J} b_{j}=k_{r, m}$, and equality (c) follows from (20).

By letting $r$ tend to infinity in both sides of (29), and substituting (30) and (31) in the RHS of (29), we get that for all $p \in \mathbb{N}$

$$
\begin{aligned}
& \lim _{r \rightarrow \infty} \mathbb{E}\left[\prod_{j=1}^{J}\left(g_{j, p}\right)^{\beta_{r, j, m}}\right] \\
& \stackrel{(\text { a) }}{=} \mathbb{E}\left[\lim _{r \rightarrow \infty} \mathbb{E}\left(\prod_{j=1}^{J}\left(g_{j, p}\right)^{\beta_{r, j, m}} \mid \sum_{j=1}^{J} \beta_{r, j, m}=k_{r, m}\right)\right] \\
& \stackrel{\text { (b) }}{=}\left[\lim _{r \rightarrow \infty} \sum_{b_{1}, \ldots, b_{J} \geq 0}\left(\begin{array}{c}
k_{r, m} \\
b_{1}, b_{2}, \ldots, b_{J}
\end{array}\right) \prod_{j=1}^{J}\left(q_{j} g_{j, p}\right)^{b_{j}}\right] \\
& =\mathbb{E}\left[\lim _{r \rightarrow \infty}\left(\sum_{j=1}^{J} q_{j} g_{j, p}\right)^{k_{r, m}}\right] \\
& \stackrel{\text { (c) }}{=} \sum_{k=1}^{d_{c, \max }}\left\{\Gamma_{k}\left(\sum_{j=1}^{J} q_{j} g_{j, p}\right)^{k}\right\}
\end{aligned}
$$




$$
=\Gamma\left(\sum_{j=1}^{J} q_{j} g_{j, p}\right)
$$

where equality (a) follows from (29) and since the right degree distribution is independent of $r$ (note that the outer expectation in equality (a) is performed w.r.t. the degree of the $m^{\text {th }}$ parity-check node); equality (b) follows from (30) and (31), and since the number of terms in the sum is bounded (this number is upper bounded by $\left(k_{r, m}\right)^{J-1}$, so it is bounded for all $r \in \mathbb{N}$ due to the fact that the maximal right degree is fixed), and equality (c) holds since the right degree distribution is independent of $r$. Since the limit in (32) does not depend on the index $m$ which appears in the inner summation at the LHS of (28) and also $\lim _{r \rightarrow \infty} n_{r}\left(1-R_{\mathrm{d}}\right)=\infty$, then we get from (32)

$$
\begin{aligned}
& \lim _{r \rightarrow \infty} \frac{1}{n_{r}\left(1-R_{\mathrm{d}}\right)} \sum_{m=1}^{n_{r}\left(1-R_{\mathrm{d}}\right)} \mathbb{E}\left[\prod_{j=1}^{J}\left(g_{j, p}\right)^{\beta_{r, j, m}}\right] \\
& \stackrel{(\text { a) }}{=} \lim _{r \rightarrow \infty} \mathbb{E}\left[\prod_{j=1}^{J}\left(g_{j, p}\right)^{\beta_{r, j, m}}\right] \\
& \stackrel{(\mathrm{b})}{=} \Gamma\left(\sum_{j=1}^{J} q_{j} g_{j, p}\right)
\end{aligned}
$$

where equality (a) follows from the fact that if $\left\{a_{r}\right\}$ is a convergent sequence then $\lim _{r \rightarrow \infty} \frac{1}{r} \sum_{i=1}^{r} a_{i}=\lim _{r \rightarrow \infty} a_{r}$, and also since any sub-sequence of a convergent sequence converges to the same limit as of the original sequence; equality (b) follows from (32). Combining (28) and (33) gives

$$
\begin{aligned}
0 \geq 1- & \sum_{j=1}^{J} p_{j} C_{j}-\left(1-R_{\mathrm{d}}\right) \\
& \cdot\left(1-\frac{1}{2 \ln 2} \sum_{p=1}^{\infty}\left\{\frac{1}{p(2 p-1)} \Gamma\left(\sum_{j=1}^{J} q_{j} g_{j, p}\right)\right\}\right) .
\end{aligned}
$$

Finally, solving the last inequality for $R_{\mathrm{d}}$ gives the upper bound on the design rate in (26).

Example 3.1: For the particular case where the $J$ parallel MBIOS channels are binary erasure channels where the erasure probability of the $j^{\text {th }}$ channel is $\varepsilon_{j}$, we get from (3)

$$
g_{j, p}=1-\varepsilon_{j}, \quad \forall j \in\{1, \ldots, J\}, \quad p \in \mathbb{N} .
$$

Since $g_{j, p}$ is independent of $p$ for a BEC, and based on the equality $\sum_{p=1}^{\infty} \frac{1}{2 p(2 p-1)}=\ln 2$, we obtain from Theorem 3.1 that the common design rate of the sequence of LDPC ensembles is upper bounded by

$$
R_{\mathrm{d}} \leq 1-\frac{\sum_{j=1}^{J} p_{j} \varepsilon_{j}}{1-\Gamma\left(1-\sum_{j=1}^{J} q_{j} \varepsilon_{j}\right)} .
$$

This result coincides with [10, Theorem 2].

The proof of Theorem 3.1 relies on the assumption that the right degree distribution $\rho$ is fixed, and does not depend on the ordinal number $r$ of the ensemble. For a capacity-achieving sequence of LDPC ensembles, both the maximal and the average right degrees tend to infinity (see [14, Theorem 1]). Hence, for a capacity-achieving sequence of LDPC codes, $\rho$ cannot be fixed.

Remark 3.1: One can think of Lemma 3.3 in terms of drawing colored balls from an urn (where the colors are determined in one to one correspondence with the assignments of the various edges to the component channels). Since an edge can only be assigned once to a channel, the balls are not returned to the urn after they are chosen. As the block length tends to infinity, so does the number of edges originating in each of the parallel channels (this is the reason for requiring that $q_{j}$ is positive for all $j$ ). Since the degree of the parity-check nodes remains finite, 
we are drawing a finite number of balls from an urn which contains an infinite number of balls of each color. Lemma 3.3 simply says that drawing without replacement is equivalent to drawing with replacement if the number of draws is finite and the number of balls of each color becomes infinite. Note that this result looks rather intuitive from a statistical point of view.

Remark 3.2: We wish to discuss a possible refinement of the statement in Theorem 3.1. Let us assume that the (overall) degree distributions $\lambda$ and $\rho$ are fixed, but due to the transmission over parallel channels, the corresponding vector of degree distributions $\underline{\lambda}_{r}=\left(\lambda_{r}^{[1]}, \ldots, \lambda_{r}^{[J]}\right)$ and also $p_{j, r}$ and $q_{j, r}$ depend on the code from the ensemble $\left(n_{r}, \lambda, \rho\right)$. Since the derivation of this theorem relies on the bounds on the conditional entropy from Section II (which are valid code by code), one can refine the statement in Theorem 3.1 so that the modified theorem permits the dependency of the vector $\left(\lambda_{r}^{[1]}, \ldots, \lambda_{r}^{[J]}\right)$ on the specific code chosen from the ensemble. In this case, the equalities in (25) are transformed to

$$
p_{j}=\lim _{r \rightarrow \infty} \mathbb{E}\left[p_{j, r}(\mathcal{C})\right], \quad q_{j}=\lim _{r \rightarrow \infty} \mathbb{E}\left[q_{j, r}(\mathcal{C})\right]
$$

where the expectation is carried over the code $\mathcal{C}$ from the ensemble $\left(n_{r}, \lambda, \rho\right)$. In this case, the proof of Theorem 3.1 involves an expectation over $\mathcal{C}$ on both sides of (27) (which is valid code by code) and then we let $r$ tend to infinity, as in (28). By invoking Jensen's inequality, Lemma 3.3 is changed under the above assumption to the inequality

$$
\begin{aligned}
& \lim _{r \rightarrow \infty} \mathbb{E}_{\mathcal{C}}\left[\frac{\left(\begin{array}{c}
\left|E_{r}^{[1]}\right| \\
b_{1}
\end{array}\right) \cdots\left(\begin{array}{c}
\left|E_{r}^{[J]}\right| \\
b_{J}
\end{array}\right)}{\left(\begin{array}{l}
\left|E_{r}\right| \\
k_{r, m}
\end{array}\right)}\right] \\
& \geq \prod_{j=1}^{J}\left(q_{j}\right)^{b_{j}} \lim _{r \rightarrow \infty}\left(\begin{array}{c}
k_{r, m} \\
b_{1}, b_{2}, \ldots, b_{J}
\end{array}\right)
\end{aligned}
$$

which holds for any set of non-negative integers $\left\{b_{1}, \ldots, b_{J}\right\}$ where $\sum_{j=1}^{J} b_{j}=k_{r, m}$. Correspondingly, (32) changes to

$$
\lim _{r \rightarrow \infty} \mathbb{E}_{\mathcal{C}}\left[\prod_{j=1}^{J}\left(g_{j, p}\right)^{\beta_{r, j, m}}\right] \geq \Gamma\left(\sum_{j=1}^{J} q_{j} g_{j, p}\right) .
$$

Therefore, from (28) and the last inequality, the upper bound on the design rate in (26) holds in the more general setting as above.

\section{Achievable Rates of Punctured LDPC Codes}

In this section we derive upper bounds on the achievable rates of punctured LDPC codes whose transmission takes place over an MBIOS channel, and the codes are ML decoded. The analysis in this section relies on the bound presented in Section III.

Let $\mathcal{C}$ be a binary linear block code. Assume that its code bits are partitioned into $J$ disjoint sets, and the bits of the $j^{\text {th }}$ set are randomly punctured with a puncturing rate $\pi_{j}$ (where $j \in\{1, \ldots, J\}$ ). The transmission of this code over an MBIOS channel is equivalent to transmitting the code over a set of $J$ parallel MBIOS channels where each of these channels forms a serial concatenation of a BEC whose erasure probability is equal to the puncturing rate $\pi_{j}$, followed by the original MBIOS channel (see e.g., [4], [9], [10], [11]).

\section{A. Some Preparatory Lemmas}

This sub-section presents two lemmas which are later used to prove results for ensembles of randomly and intentionally punctured LDPC codes (denoted by RP-LDPC and IP-LDPC codes, respectively).

In the following lemma, we consider a punctured linear block code and provide an upper bound on the conditional entropy of a codeword before puncturing, given the received sequence at the output of the channel. This upper bound is expressed in terms of the bit error probability of the punctured code.

Lemma 4.1: Let $\mathcal{C}^{\prime}$ be a binary linear block code of length $n$ and rate $R^{\prime}$, and let $\mathcal{C}$ be a code which is obtained from $\mathcal{C}^{\prime}$ by puncturing some of its code bits. Assume that the transmission of the code $\mathcal{C}$ takes place over an arbitrary communication channel, and the code is decoded by an arbitrary decoding algorithm. Let $\mathbf{X}^{\prime}=\left(X_{1}^{\prime}, \ldots, X_{n}^{\prime}\right)$ and $\mathbf{Y}=\left(Y_{1}, \ldots, Y_{n}\right)$ (where the punctured bits are replaced by question marks which have an LLR of zero) designate 
the transmitted codeword of $\mathcal{C}^{\prime}$ and the received sequence, respectively. Then, the conditional entropy of the original codeword of $\mathcal{C}^{\prime}$ given the received sequence satisfies

$$
\frac{H\left(\mathbf{X}^{\prime} \mid \mathbf{Y}\right)}{n} \leq R^{\prime} h_{2}\left(P_{\mathrm{b}}\right)
$$

where $P_{\mathrm{b}}$ designates the bit error probability of the punctured code $\mathcal{C}$.

Proof: The proof follows directly from Lemma 2.2, and the equivalence between the transmission of punctured codes over an MBIOS channel and the special case of transmitting these codes over a set of parallel channels (see the introductory paragraph of Section IV).

Puncturing serves to increase the rate of the original code by reducing the length of the codeword. It may however cause several codewords to be mapped onto a single codeword, thereby reducing the dimension of the code. Consider a binary linear code, $\mathcal{C}^{\prime}$, of length $n$ and rate $R^{\prime}$ and assume a fraction $\gamma$ of its code bits are punctured. In the case that the dimension is not reduced by puncturing, the rate of the punctured code is given by $R=\frac{R^{\prime}}{1-\gamma}$. In the general case, we cannot guarantee that the dimension of the code is not reduced. However, for a sequence of punctured codes whose bit error probability vanishes as the block length of the codes tends to infinity, the following lemma shows that the rate of the punctured codes converges to the desired rate $R$.

Lemma 4.2: Let $\left\{\mathcal{C}_{r}^{\prime}\right\}$ be a sequence of binary linear block codes of length $n_{r}$ and rate $R_{r}^{\prime}$, and let $\left\{\mathcal{C}_{r}\right\}$ be a sequence of codes which is obtained from $\left\{\mathcal{C}_{r}^{\prime}\right\}$ by puncturing a fraction $\gamma$ of the code bits. Assume the sequence of punctured codes $\left\{\mathcal{C}_{r}\right\}$ achieves vanishing bit error probability in the limit where we let $r$ tend to infinity. Then, the asymptotic rate $R$ of the sequence of punctured codes is given by

$$
R=\frac{R^{\prime}}{1-\gamma}
$$

where $R^{\prime}=\lim _{r \rightarrow \infty} R_{r}^{\prime}$ is the asymptotic rate of the original sequence of codes $\left\{\mathcal{C}_{r}^{\prime}\right\}$.

Proof: Let $\mathbf{X}_{r}^{\prime}=\left(X_{1}^{\prime}, \ldots, X_{n_{r}}^{\prime}\right)$ and $\mathbf{Y}_{r}=\left(Y_{r} \ldots, Y_{n_{r}}\right)$ designate the original codeword (before puncturing) and the received sequence (after puncturing), respectively. Since we assume the there exists a decoding algorithm such that the punctured codes achieve vanishing bit error probability, we have from lemma 4.1 that

$$
\lim _{r \rightarrow \infty} \frac{H\left(\mathbf{X}_{r}^{\prime} \mid \mathbf{Y}_{r}\right)}{n_{r}}=0
$$

Let $\mathbf{X}_{r}=\left(X_{1}, \ldots, X_{n_{r}}\right)$ designate the codeword after puncturing (where the punctured bits are replaced by question marks). Since $\mathbf{X}_{r}^{\prime} \Rightarrow \mathbf{X}_{r} \Rightarrow \mathbf{Y}_{r}$ forms a Markov chain, then by the information processing inequality, we get $H\left(\mathbf{X}_{r}^{\prime} \mid \mathbf{X}_{r}\right) \leq H\left(\mathbf{X}_{r}^{\prime} \mid \mathbf{Y}_{r}\right)$. The non-negativity of the conditional entropy therefore yields that

$$
\lim _{r \rightarrow \infty} \frac{H\left(\mathbf{X}_{r}^{\prime} \mid \mathbf{X}_{r}\right)}{n_{r}}=0 .
$$

Denote the dimensions of the codes $\mathcal{C}_{r}^{\prime}$ and $\mathcal{C}_{r}$ by $d_{r}^{\prime}$ and $d_{r}$, respectively. Since $\mathcal{C}_{r}^{\prime}$ is binary and linear, every codeword of $\mathcal{C}_{r}$ originates from exactly $2^{d_{r}^{\prime}-d_{r}}$ different codewords of $\mathcal{C}_{r}^{\prime}$. The codewords are assumed to be transmitted with equal probability, and therefore $H\left(\mathbf{X}_{r}^{\prime} \mid \mathbf{X}_{r}\right)=d_{r}^{\prime}-d_{r}$. Let $R_{r}$ designate the rate of the punctured code $\mathcal{C}_{r}$. By definition, $d_{r}^{\prime}=R_{r}^{\prime} n_{r}$, and since $n_{r}(1-\gamma)$ forms the block length of the punctured code $\mathcal{C}_{r}$, then $d_{r}=R_{r} n_{r}(1-\gamma)$. Substituting the last three equalities into (38) gives

$$
\lim _{r \rightarrow \infty}\left(R_{r}^{\prime}-R_{r}(1-\gamma)\right)=0
$$

This completes the proof of the lemma.

For a sequence of codes $\left\{\mathcal{C}_{r}^{\prime}\right\}$, it is natural to refer to their code rates $R_{r}^{\prime}$. However, for sequences of ensembles, where parity-check matrices are randomly picked, such matrices are unlikely to be full rank. Hence, a more natural approach is to refer to their design rates. To this end, we define the design rate of codes which are obtained by puncturing some code bits of binary linear block codes.

Definition 4.1: Let $\mathcal{C}^{\prime}$ be a binary linear block code of length $n, H^{\prime}$ be a $c \times n$ parity-check matrix of $\mathcal{C}^{\prime}$ and $R_{d}^{\prime} \triangleq 1-\frac{c}{n}$ designate the design rate of the code $\mathcal{C}^{\prime}$. Let $\mathcal{C}$ be a code which is obtained from $\mathcal{C}^{\prime}$ by puncturing a fraction $\gamma$ of the code bits. The design rate of $\mathcal{C}$ is defined as

$$
R_{\mathrm{d}} \triangleq \frac{R_{\mathrm{d}}^{\prime}}{1-\gamma}
$$


From Lemma 4.2, it follows that for an arbitrary sequence of punctured codes which achieves vanishing bit error probability, their asymptotic design rate is equal in probability 1 to their asymptotic rate if and only if this condition also holds for the original sequence of codes before their puncturing. For un-punctured ensembles of LDPC codes, a sufficient condition for the asymptotic convergence of the rate to the design rate is introduced in [8, Lemma 7] (which is also presented in the preliminaries of our companion paper as [17, Lemma 2.1]). In Section IV-D, we apply this lemma to show that the bounds on the achievable rates of ensembles of punctured LDPC codes apply to their actual code rates and not only to their asymptotic design rates.

\section{B. Randomly Punctured LDPC Codes}

In this section, we consider the achievable rates of randomly punctured LDPC (RP-LDPC) codes. We assume that the transmission of these codes takes place over an MBIOS channel, and refer to their achievable rates under optimal ML decoding. The upper bound on the achievable rates of ensembles of RP-LDPC codes relies on the analysis in Section III where we derived an upper bound on the achievable rates of LDPC codes for parallel channels.

In the following, we assume that the communication takes place over an MBIOS channel with capacity $C$, and define

$$
g_{p} \triangleq \int_{0}^{\infty} a(l)\left(1+e^{-l}\right) \tanh ^{2 p}\left(\frac{l}{2}\right) \mathrm{d} l, \quad p \in \mathbb{N}
$$

where $a$ designates the pdf of the LLR of the channel given that its input is zero.

Theorem 4.1: Let $\left\{\left(n_{r}, \lambda, \rho\right)\right\}_{r=1}^{\infty}$ be a sequence of ensembles of binary LDPC codes whose block length $\left(n_{r}\right)$ tends to infinity as $r \rightarrow \infty$. Assume that a sequence of ensembles of RP-LDPC codes is constructed in the following way: for each code from an ensemble of the original sequence, a subset of $\alpha n_{r}$ code bits is a-priori selected, and these bits are randomly punctured at a fixed rate $\left(P_{\mathrm{pct}}\right)$. Assume that the punctured codes are transmitted over an MBIOS channel with capacity $C$, and that in the limit where $r$ approaches infinity, the sequence of ensembles of RP-LDPC codes achieves vanishing bit error probability under some decoding algorithm. Then in probability 1 w.r.t. the random puncturing patterns, the asymptotic design rate $\left(R_{\mathrm{d}}\right)$ of the new sequence satisfies

$$
\begin{aligned}
R_{\mathrm{d}} \leq & \frac{1}{1-\alpha P_{\mathrm{pct}}} \\
& \cdot\left(\begin{array}{l}
\left.1-\frac{1-\left(1-\alpha P_{\mathrm{pct}}\right) C}{1-\frac{1}{2 \ln 2} \sum_{p=1}^{\infty} \frac{\Gamma\left(\left(1-P_{\mathrm{pct}}+\xi\right) g_{p}\right)}{p(2 p-1)}}\right)
\end{array}\right.
\end{aligned}
$$

where $\Gamma$ denotes the right degree distribution (from the node perspective) of the original sequence, $g_{p}$ is introduced in (40), and $\xi$ is the following positive number:

$$
\xi \triangleq 2(1-\alpha) P_{\mathrm{pct}} \int_{0}^{1} \lambda(x) \mathrm{d} x .
$$

Proof: By assumption, we select a set of code bits whose size is a fraction $\alpha$ of the $n_{r}$ code bits, and these bits are randomly punctured at rate $P_{\text {pct }}$. The transmission of the resulting codeword over an MBIOS channel is equivalent to the transmission of the original codeword over a set of $J=2$ parallel channels. The first channel, referring to the set of code bits which are randomly punctured, is a serial concatenation of a BEC with erasure probability $P_{\mathrm{pct}}$ and the original MBIOS channel; the second channel which refers to the rest of the bits (which are transmitted without being randomly punctured) is the original MBIOS channel. For simplicity, let us first assume that the degree distribution associated with the selected subset of $\alpha n_{r}$ code bits which are randomly punctured is independent of the specific code from the ensemble $\left(n_{r}, \lambda, \rho\right)$. Based on the discussion above and the notation in Section III, the transmission of the $n_{r}$ code bits over these two parallel channels induces a sequence of ensembles of LDPC codes, $\left\{\left(n_{r}, \underline{\lambda}_{r}, \rho\right)\right\}_{r=1}^{\infty}$, where $\underline{\lambda}_{r}=\left(\lambda_{r}^{[1]}, \lambda_{r}^{[2]}\right)$ depends on the selection of the subset of $\alpha n_{r}$ code bits 
which are randomly punctured. Following this equivalence, we get from the notation in Theorem 3.1 that

$$
\begin{aligned}
& p_{1}=\alpha, \quad p_{2}=1-\alpha, \quad C_{1}=C\left(1-P_{\mathrm{pct}}\right), \quad C_{2}=C \\
\Rightarrow & \sum_{j=1}^{J} p_{j} C_{j}=C\left(1-\alpha P_{\mathrm{pct}}\right) .
\end{aligned}
$$

In order to apply Theorem 3.1 to our case, we find a global lower bound on the sum $\sum_{j=1}^{J} q_{j} g_{j, p}$ which does not depend on the a-priori selection of the subset of randomly punctured code bits. From (3) and (40), it follows that for all $p \in \mathbb{N}$ :

$$
\begin{aligned}
g_{1, p} & =\int_{0}^{\infty}\left[P_{\mathrm{pct}} \delta(l)+\left(1-P_{\mathrm{pct}}\right) a(l)\right]\left(1+e^{-l}\right) \tanh ^{2 p}\left(\frac{l}{2}\right) \mathrm{d} l \\
& =\left(1-P_{\mathrm{pct}}\right) \int_{0}^{\infty} a(l)\left(1+e^{-l}\right) \tanh ^{2 p}\left(\frac{l}{2}\right) \mathrm{d} l \\
& =\left(1-P_{\mathrm{pct}}\right) g_{p}
\end{aligned}
$$

and $g_{2, p}=g_{p}$. Based on Lemmas 3.1 and 3.2, we get that for all $p \in \mathbb{N}$

$$
\begin{aligned}
& q_{1} g_{1, p}+q_{2} g_{2, p} \\
& =\frac{\alpha g_{p}\left(1-P_{\mathrm{pct}}\right) \int_{0}^{1} \lambda(x) \mathrm{d} x}{\int_{0}^{1} \lambda_{r}^{[1]}(x) \mathrm{d} x}+\frac{(1-\alpha) g_{p} \int_{0}^{1} \lambda(x) \mathrm{d} x}{\int_{0}^{1} \lambda_{r}^{[2]}(x) \mathrm{d} x}
\end{aligned}
$$

where the following constraint is satisfied (see (22) and (43)):

$$
\frac{\alpha}{\int_{0}^{1} \lambda_{r}^{[1]}(x) \mathrm{d} x}+\frac{1-\alpha}{\int_{0}^{1} \lambda_{r}^{[2]}(x) \mathrm{d} x}=\frac{1}{\int_{0}^{1} \lambda(x) \mathrm{d} x}
$$

and

$$
\int_{0}^{1} \lambda_{r}^{[1]}(x) \mathrm{d} x \leq \frac{1}{2}, \quad \int_{0}^{1} \lambda_{r}^{[2]}(x) \mathrm{d} x \leq \frac{1}{2}
$$

due to the fact that $\lambda_{r}^{[1]}(x) \leq x$ and $\lambda_{r}^{[2]}(x) \leq x$ for $x \in[0,1]$ (even without explicitly knowing $\lambda_{r}^{[1]}$ and $\lambda_{r}^{[2]}$ which depend on the a-priori choice of the subset of bits which are randomly punctured). Based on (44)-(46), we get

$$
\begin{aligned}
& q_{1} g_{1, p}+q_{2} g_{2, p} \\
& =\left(1-P_{\mathrm{pct}}\right) g_{p} \int_{0}^{1} \lambda(x) \mathrm{d} x \cdot\left(\frac{\alpha}{\int_{0}^{1} \lambda_{r}^{[1]}(x) \mathrm{d} x}+\frac{1-\alpha}{\int_{0}^{1} \lambda_{r}^{[2]}(x) \mathrm{d} x}\right) \\
& \quad+\frac{(1-\alpha) P_{\mathrm{pct}} g_{p} \int_{0}^{1} \lambda(x) \mathrm{d} x}{\int_{0}^{1} \lambda_{r}^{[2]}(x) \mathrm{d} x} \\
& =\left(1-P_{\mathrm{pct}}\right) g_{p}+\frac{(1-\alpha) P_{\mathrm{pct}} g_{p} \int_{0}^{1} \lambda(x) \mathrm{d} x}{\int_{0}^{1} \lambda_{r}^{[2]}(x) \mathrm{d} x} \\
& \geq\left(1-P_{\mathrm{pct}}+2(1-\alpha) P_{\mathrm{pct}} \int_{0}^{1} \lambda(x) \mathrm{d} x\right) g_{p} \\
& =\left(1-P_{\mathrm{pct}}+\xi\right) g_{p}
\end{aligned}
$$


where $\xi$ is defined in (42). Since the degree distribution $\Gamma$ is a monotonic increasing function, then

$$
\Gamma\left(\sum_{j=1}^{J} q_{j} g_{j, p}\right) \geq \Gamma\left(\left(1-P_{\mathrm{pct}}+\xi\right) g_{p}\right) .
$$

By substituting (43) and (47) in the RHS of (26), we obtain the following upper bound on the asymptotic design rate of the original sequence

$$
R_{\mathrm{d}}^{\prime} \leq 1-\frac{1-\left(1-\alpha P_{\mathrm{pct}}\right) C}{1-\frac{1}{2 \ln 2} \sum_{p=1}^{\infty}\left\{\frac{1}{p(2 p-1)} \Gamma\left(\left(1-P_{\mathrm{pct}}+\xi\right) g_{p}\right)\right\}} .
$$

Since as $r \rightarrow \infty$, in probability 1 w.r.t. the puncturing patterns, a fraction $\gamma=\alpha P_{\text {pct }}$ of the code bits are punctured, then the asymptotic design rate $\left(R_{\mathrm{d}}\right)$ of this sequence satisfies the equality

$$
R_{\mathrm{d}}=\frac{R_{\mathrm{d}}^{\prime}}{1-\alpha P_{\mathrm{pct}}}
$$

from which the theorem follows.

For the case where the degree distribution associated with the subset of code bits which are randomly punctured depends on the code $\mathcal{C}$ from the ensemble $\left(n_{r}, \lambda, \rho\right)$, the pair $\left(\lambda_{r}^{[1]}, \lambda_{r}^{[2]}\right)$ cannot be considered to be uniform over all the codes from this ensemble. In this case, Theorem 3.1 is not directly applicable. In order to circumvent the problem, we rely on the discussion in Remark 3.2, and on the fact that the lower bound on $q_{1} g_{1, p}+q_{2} g_{2, p}$ which is given above in terms of $\xi$ from (42) is universal for all the codes from this ensemble (i.e., it only depends on $\lambda$, but does not depend on the specific degree distributions $\lambda_{r}^{[1]}(\mathcal{C})$ and $\lambda_{r}^{[2]}(\mathcal{C})$ which are associated with the code $\mathcal{C}$ from the ensemble). In light of this reasoning, the proof of the theorem for ensembles of RP-LDPC codes also follows in the more general setting where the degree distribution associated with the subset of the code bits which are randomly punctured depends on the specific code from the ensemble.

Remark 4.1: Note that in the above proof, we derive an upper bound on the number of edges adjacent to variable nodes which are punctured in probability $P_{\text {pct }}$; this is done by assuming that the degree of all the un-punctured nodes is 2 (which is the minimal possible degree for a variable node), and counting the number of the remaining edges. In the case that the original codes before puncturing have a minimal variable degree of $\Lambda_{\min }>2$, the upper bound can be tightened by assuming that each un-punctured node is of degree $\Lambda_{\min }$. This results in replacing $\xi$ in (42) with $\xi^{\prime} \triangleq \Lambda_{\min }(1-\alpha) P_{\text {pct }} \int_{0}^{1} \lambda(x) \mathrm{d} x$.

\section{Intentionally Punctured LDPC Codes}

In [4], Ha and McLaughlin show that good codes can be constructed by puncturing good ensembles of LDPC codes using a technique called "intentional puncturing". In this approach, the code bits are partitioned into disjoint sets so that each set contains all the code bits whose corresponding variable nodes have the same degree. The code bits in each of these sets are randomly punctured at a fixed puncturing rate.

We briefly present the notation used in [4] for the characterization of ensembles of intentionally punctured LDPC (IP-LDPC) codes. Consider an ensemble of LDPC codes with left and right edge degree distributions $\lambda$ and $\rho$, respectively. For each degree $j$ such that $\lambda_{j}>0$, a puncturing rate $\pi_{j} \in[0,1]$ is determined for randomly puncturing the set of code bits which correspond to variable nodes of degree $j$. The polynomial associated with this puncturing pattern is

$$
\pi^{(0)}(x) \triangleq \sum_{j=1}^{\infty} \pi_{j} x^{j-1} .
$$

An ensemble of IP-LDPC codes can be therefore represented by the quadruplet $\left(n, \lambda, \rho, \pi^{(0)}\right)$ where $n$ designates the block length of these codes, $\lambda$ and $\rho$ are the left and right degree distributions from the edge perspective, respectively, and $\pi^{(0)}$ is the polynomial which corresponds to the puncturing pattern, as given in (49). The average fraction of punctured bits is given by $p^{(0)}=\sum_{j=1}^{\infty} \Lambda_{j} \pi_{j}$ where $\Lambda$ is the left node degree distribution of the original LDPC ensemble. The following statement, which relies on Theorem 3.1, provides an upper bound on the common 
design rate of a sequence of ensembles of IP-LDPC codes. This bound refers to ML decoding (and hence, it also holds for any sub-optimal decoding algorithm).

Theorem 4.2: Let $\left\{\left(n_{r}, \lambda, \rho, \pi^{(0)}\right)\right\}_{r=1}^{\infty}$ be a sequence of ensembles of IP-LDPC codes transmitted over an MBIOS channel, and assume that $n_{r}$ tends to infinity as $r \rightarrow \infty$. Let $C$ be the channel capacity, and $a$ be the pdf of the LLR at the output of the channel given its input is zero. If the asymptotic bit error probability of this sequence vanishes under ML decoding (or any sub-optimal decoding algorithm) as $r \rightarrow \infty$, then in probability 1 w.r.t. the puncturing patterns, the asymptotic design rate $R_{\mathrm{d}}$ of these ensembles satisfies

$$
\begin{aligned}
R_{\mathrm{d}} & \leq \frac{1}{1-p^{(0)}} \\
& \cdot\left[1-\frac{1-\left(1-p^{(0)}\right) C}{1-\frac{1}{2 \ln 2} \sum_{p=1}^{\infty}\left\{\frac{1}{p(2 p-1)} \Gamma\left(\left(1-\sum_{j=1}^{\infty} \lambda_{j} \pi_{j}\right) g_{p}\right)\right\}}\right]
\end{aligned}
$$

where $\Gamma$ denotes the right degree distribution from the node perspective,

$$
p^{(0)} \triangleq \sum_{j=1}^{\infty} \Lambda_{j} \pi_{j}
$$

designates the average puncturing rate of the code bits, and $g_{p}$ is the functional of the MBIOS channel introduced in (40).

Proof: The proof follows from Theorem 3.1, and the observation that IP-LDPC codes form a special case of the ensemble $(n, \underline{\lambda}, \rho)$ examined in Section III. For a sequence of ensembles of IP-LDPC codes, $\left\{\left(n_{r}, \lambda, \rho, \pi^{(0)}\right)\right\}$, the number of parallel MBIOS channels used for transmission is equal to the number of strictly positive coefficients in the polynomial $\lambda$, i.e., $J \triangleq\left|\left\{i: \lambda_{i}>0\right\}\right|$. Denote these degrees by $i_{1}, \ldots, i_{J}$, then the bits transmitted over the $j^{\text {th }}$ channel are those involved in exactly $i_{j}$ parity-check equations (i.e., the bits whose corresponding variable nodes are of degree $i_{j}$ ). From the above discussion, it follows that the fraction of code bits transmitted over the $j^{\text {th }}$ channel is given by

$$
p_{j}=\Lambda_{i_{j}}, \quad j \in\{1, \ldots, J\}
$$

and the fraction of edges in the bipartite graph which are connected to variable nodes transmitted of the $j^{\text {th }}$ channel is given by

$$
q_{j}=\lambda_{i_{j}}, \quad j \in\{1, \ldots, J\} .
$$

The transmission of IP-LDPC codes over an MBIOS channel is equivalent to transmitting the original codes (before puncturing) over a set of $J$ parallel MBIOS channels where each of these channels is formed by a serial concatenation of a BEC whose erasure probability is equal to the puncturing rate $\pi_{i_{j}}$, followed by the original MBIOS channel. Hence, the pdf of the LLR at the output of the $j^{\text {th }}$ MBIOS channel, given its symbol input is zero, gets the form

$$
a(l ; j)=\pi_{i_{j}} \delta_{0}(l)+\left(1-\pi_{i_{j}}\right) a(l), \quad l \in \mathbb{R}
$$

and the capacity of this channel is

$$
C_{j}=\left(1-\pi_{i_{j}}\right) C .
$$

By substituting (54) into (3), we get that for all $j \in\{1, \ldots, J\}$ and $p \in \mathbb{N}$

$$
\begin{aligned}
g_{j, p} & =\int_{0}^{\infty}\left[\pi_{i_{j}} \delta_{0}(l)+\left(1-\pi_{i_{j}}\right) a(l)\right]\left(1+e^{-l}\right) \tanh ^{2 p}\left(\frac{l}{2}\right) \mathrm{d} l \\
& =\left(1-\pi_{i_{j}}\right) \int_{0}^{\infty} a(l)\left(1+e^{-l}\right) \tanh ^{2 p}\left(\frac{l}{2}\right) \mathrm{d} l \\
& =\left(1-\pi_{i_{j}}\right) g_{p}
\end{aligned}
$$

where the last equality is based on (40). The statement now follows by substituting (52), (53), (55) and (56) in (26); we use the scaling factor for the design rate of the punctured codes, as given in Definition 4.1. In this case, the 
parameter $\gamma$ tends to the average puncturing rate $p^{(0)}$ of the code bits, as defined in (51), where this convergence is in probability 1 w.r.t. the puncturing patterns. Finally, since $\lambda_{j}=\Lambda_{j}=0$ for $j \notin\left\{i_{1}, \ldots, i_{J}\right\}$, then regarding the sums in the RHS of (50), we get the equalities $\sum_{j=1}^{\infty} \Lambda_{j} \pi_{j}=\sum_{j=1}^{J} \Lambda_{i_{j}} \pi_{i_{j}}$ and $\sum_{j=1}^{\infty} \lambda_{j} \pi_{j}=\sum_{j=1}^{J} \lambda_{i_{j}} \pi_{i_{j}}$. This completes the proof of the theorem.

Remark 4.2: Let us consider a more general case of punctured ensembles of LDPC codes where the original code bits are split into $J$ arbitrary sets and each set is punctured at a different rate. For this general case, it is possible to apply Theorem 3.1 to derive an upper bound on the achievable rates which only depends on the expected fractions of punctured code bits and edges in the graph attached to variable nodes of punctured bits. Theorems 4.1 and 4.2 emerge as corollaries of such a theorem (in this paper we do not take this approach since we analyze two strategies of puncturing as special cases of transmission over parallel channels). In the case of ensembles of RP-LDPC codes, the fraction of edges adjacent to punctured bits is not known in general. Hence, for the derivation of upper bounds on their achievable rates, we employ an upper bound on the fraction of edges adjacent to punctured bits in a similar way to the proof of Theorem 4.1 .

\section{Numerical Results for Intentionally Punctured LDPC Codes}

In this section, we present a comparison between thresholds under MPI decoding and bounds on thresholds under ML decoding for ensembles of IP-LDPC codes. It is assumed that the transmission of the punctured LDPC codes takes place over a binary-input AWGN channel. The pairs of degree distributions and the corresponding puncturing patterns were originally presented in [4], [5]. We study the inherent gap to capacity and consider how close to optimal iterative decoding is for these ensembles (in the asymptotic case where the block length goes to infinity).

We refer here to three ensembles of IP-LDPC codes: Tables I and II refer to two ensembles of rate $-\frac{1}{2}$ LDPC codes which by puncturing, their rates vary between 0.50 and 0.91 ; Table III refers to an ensemble of rate $-\frac{1}{10}$ LDPC codes which by puncturing, its rate varies between 0.10 and 0.83 . Based on [8, Lemma 7], we verify that the design rates of these three ensembles of LDPC codes (before puncturing) are equal in probability 1 to the asymptotic rates of codes from these ensembles. This conclusion still holds for the punctured LDPC ensembles given in Tables I-III (see Lemma 4.2). This enables to calculate the capacity limits which refer to the design rates of these ensembles, and to evaluate the gaps to capacity under ML decoding and iterative decoding for these ensembles of punctured LDPC codes.

For various ensembles of IP-LDPC codes, Tables I-III provide lower bounds on the inherent gap to capacity under optimal ML decoding (based on Theorem 4.2); these values are compared to the corresponding gaps to capacity under MPI decoding (whose calculation is based on the density evolution analysis). On one hand, Tables I-III provide a quantitative assessment of the loss in the asymptotic performance which is attributed to the sub-optimality of iterative decoding (as compared to ML decoding), and on the other hand, these tables provide an assessment of the inherent loss in performance which is attributed to the structure of the ensembles, even if ML decoding could be applied to decode these codes. The loss in performance in both cases is measured in terms of $\frac{E_{\mathrm{b}}}{N_{0}}$ in decibels. It is demonstrated in Tables I-III that for various good ensembles of IP-LDPC codes, the asymptotic loss in performance due to the code structure is non-negligible as compared to the corresponding loss due to the sub-optimality of iterative decoding. As an example, for all the ensembles of IP-LDPC codes considered in Table I (which were originally introduced in [4, Table 2]), the gap to capacity under the sum-product iterative decoding algorithm does not exceed $0.6 \mathrm{~dB}$; however, under ML decoding, the gap to capacity is always greater than $\frac{1}{3}$ of the corresponding gap to capacity under this iterative decoding algorithm; therefore, the results in Table I regarding the thresholds under ML decoding further emphasize the efficiency of the sum-product decoding algorithm for these ensembles, especially in light of its moderate complexity.

Tables I-III also show that the performance of the punctured LDPC codes is degraded at high rates, where one needs to pay a considerable penalty for using punctured codes. This phenomenon was explained in [11, Theorem 1] by the threshold effect for ensembles of punctured LDPC codes.

Following the performance analysis of punctured LDPC codes in [2], [4], [5], [11], the numerical results shown in Tables I-III exemplify the high potential of puncturing in designing codes which operate closely to the Shannon capacity limit and are used for rate-compatible coding for various MBIOS channels. Other examples of capacityachieving ensembles of punctured codes on graphs are the irregular repeat-accumulate (IRA) codes and accumulaterepeat-accumulate (ARA) codes. Recently, it was shown by Pfister et al. that properly designed nonsystematic IRA 
TABLE I

COMPARISON OF THRESHOLDS FOR ENSEMBLES OF IP-LDPC CODES WHERE THE ORIGINAL ENSEMBLE BEFORE PUNCTURING HAS THE DEGREE DISTRIBUTIONS $\lambda(x)=0.25105 x+0.30938 x^{2}+0.00104 x^{3}+0.43853 x^{9}$ AND $\rho(x)=0.63676 x^{6}+0.36324 x^{7}$ (SO ITS DESIGN RATE IS $\frac{1}{2}$ ). THE TRANSMISSION OF THESE CODES TAKES PLACE OVER A BINARY-INPUT AWGN CHANNEL. THE TABLE COMPARES VALUES OF $\frac{E_{\mathrm{b}}}{N_{0}}$ REFERRING TO THE CAPACITY LIMIT, THE BOUND GIVEN IN THEOREM 4.2 (WHICH PROVIDES A LOWER BOUND ON THE $\frac{E_{\mathrm{b}}}{N_{0}}$-THRESHOLD UNDER ML DECODING), AND THRESHOLDS UNDER ITERATIVE DECODING. THE FRACTIONAL GAP TO CAPACITY IN THE RIGHTMOST COLUMN MEASURES THE RATIO OF THE GAP TO CAPACITY UNDER ML DECODING AND THE ACHIEVABLE GAP TO CAPACITY UNDER ITERATIVE DECODING. THE DEGREE DISTRIBUTIONS FOR THE ENSEMBLE OF LDPC CODES, AND THE POLYNOMIALS WHICH CORRESPOND TO ITS PUNCTURING PATTERNS ARE GIVEN IN [4, TABLE 2].

\begin{tabular}{|c|c|c|c|c|c|}
\hline \multicolumn{1}{|c|}{$\begin{array}{c}\pi^{(0)}(x) \\
\text { (puncturing pattern) }\end{array}$} & $\begin{array}{c}\text { Design } \\
\text { rate }\end{array}$ & $\begin{array}{c}\text { Capacity } \\
\text { limit }\end{array}$ & $\begin{array}{c}\text { Lower bound } \\
\text { (ML decoding) }\end{array}$ & $\begin{array}{c}\text { Iterative (IT) } \\
\text { Decoding }\end{array}$ & $\begin{array}{c}\text { Fractional gap to } \\
\text { capacity (ML vs. IT) }\end{array}$ \\
\hline \hline \multicolumn{1}{c}{0} & 0.500 & $0.187 \mathrm{~dB}$ & $0.270 \mathrm{~dB}$ & $0.393 \mathrm{~dB}$ & $\geq 40.3 \%$ \\
\hline $\begin{array}{l}0.07886 x+0.01405 x^{2}+ \\
0.06081 x^{3}+0.07206 x^{9}\end{array}$ & 0.528 & $0.318 \mathrm{~dB}$ & $0.397 \mathrm{~dB}$ & $0.526 \mathrm{~dB}$ & $\geq 37.9 \%$ \\
\hline $\begin{array}{l}0.20276 x+0.09305 x^{2}+ \\
0.03356 x^{3}+0.16504 x^{9}\end{array}$ & 0.592 & $0.635 \mathrm{~dB}$ & $0.716 \mathrm{~dB}$ & $0.857 \mathrm{~dB}$ & $\geq 36.4 \%$ \\
\hline $\begin{array}{l}0.25381 x+0.15000 x^{2}+ \\
0.34406 x^{3}+0.019149 x^{9}\end{array}$ & 0.629 & $0.836 \mathrm{~dB}$ & $0.923 \mathrm{~dB}$ & $1.068 \mathrm{~dB}$ & $\geq 37.3 \%$ \\
\hline $\begin{array}{l}0.31767 x+0.18079 x^{2}+ \\
0.05265 x^{3}+0.24692 x^{9}\end{array}$ & 0.671 & $1.083 \mathrm{~dB}$ & $1.171 \mathrm{~dB}$ & $1.330 \mathrm{~dB}$ & $\geq 35.6 \%$ \\
\hline $\begin{array}{l}0.36624 x+0.24119 x^{2}+ \\
0.49649 x^{3}+0.27318 x^{9}\end{array}$ & 0.719 & $1.398 \mathrm{~dB}$ & $1.496 \mathrm{~dB}$ & $1.664 \mathrm{~dB}$ & $\geq 36.9 \%$ \\
\hline $\begin{array}{l}0.41838 x+0.29462 x^{2}+ \\
0.05265 x^{3}+0.30975 x^{9}\end{array}$ & 0.774 & $1.814 \mathrm{~dB}$ & $1.927 \mathrm{~dB}$ & $2.115 \mathrm{~dB}$ & $\geq 37.2 \%$ \\
\hline $\begin{array}{l}0.47074 x+0.34447 x^{2}+ \\
0.02227 x^{3}+0.34997 x^{9}\end{array}$ & 0.838 & $2.409 \mathrm{~dB}$ & $2.547 \mathrm{~dB}$ & $2.781 \mathrm{~dB}$ & $\geq 37.1 \%$ \\
\hline $\begin{array}{l}0.52325 x+0.39074 x^{2}+ \\
0.01324 x^{3}+0.39436 x^{9}\end{array}$ & 0.912 & $3.399 \mathrm{~dB}$ & $3.607 \mathrm{~dB}$ & $3.992 \mathrm{~dB}$ & $\geq 35.1 \%$ \\
\hline
\end{tabular}

codes achieve the capacity of the BEC with bounded decoding complexity per information bit [9]. This bounded complexity result is achieved by puncturing all the information bits of the IRA codes, and allowing in this way a sufficient number of state nodes in the Tanner graph representing the codes. This is in contrast to all previous constructions of capacity-achieving LDPC codes which refer to bipartite graphs without state nodes and whose complexity becomes unbounded as their gap to capacity vanishes (for an information-theoretic proof which explains why the complexity becomes unbounded in this case, the reader is referred to [14, Theorem 2.1]). The decoding complexity of punctured LDPC codes for parallel channels is addressed in the next section.

\section{Lower Bounds on the Decoding Complexity of LDPC Codes For PARAllel Channels}

The scope of this section is to derive a lower bound on the decoding complexity (per iteration) of LDPC codes transmitted over parallel MBIOS channels. The lower bound holds under MPI decoding, and it grows like the logarithm of the inverse of the gap (in rate) to capacity. Interestingly, a logarithmic behavior of the parity-check density (which forms a measure of the decoding complexity per iteration) in terms of the gap to capacity also characterizes the upper bound derived in [3, Section 3]; this upper bound refers to MacKay's ensemble of LDPC codes whose transmission takes place over a set of parallel MBIOS channels.

In the previous section we regarded the transmission of punctured LDPC codes over MBIOS channels as a special case of the transmission of the original codes (before puncturing) over a set of parallel MBIOS channels. Hence, the aforementioned bound is later applied to obtain lower bounds on the decoding complexity of punctured LDPC codes. This section refers to an appendix which suggests a simplified re-derivation of [9, Theorems 3 and 4], and shows that the bounds introduced in this section are tighter. 
TABLE II

COMPARISON OF THRESHOLDS FOR ENSEMBLES OF IP-LDPC CODES WHERE THE ORIGINAL LDPC ENSEMBLE BEFORE PUNCTURING HAS THE DEGREE DISTRIBUTIONS $\lambda(x)=0.23403 x+0.21242 x^{2}+0.14690 x^{5}+0.10284 x^{6}+0.30381 x^{19}$ AND $\rho(x)=0.71875 x^{7}+0.28125 x^{8}$ (SO ITS DESIGN RATE IS $\frac{1}{2}$ ). THE TRANSMISSION OF THESE CODES TAKES PLACE OVER A BINARY-INPUT AWGN CHANNEL. THE TABLE COMPARES VALUES OF $\frac{E_{\mathrm{b}}}{N_{0}}$ REFERRING TO THE CAPACITY LIMIT, THE BOUND GIVEN IN THEOREM 4.2 (WHICH PROVIDES A LOWER BOUND ON THE $\frac{E_{\mathrm{b}}}{N_{0}}$-THRESHOLD UNDER ML DECODING), AND THRESHOLDS UNDER ITERATIVE DECODING. THE FRACTIONAL GAP TO CAPACITY IN THE RIGHTMOST COLUMN MEASURES THE RATIO OF THE GAP TO CAPACITY UNDER ML DECODING AND THE ACHIEVABLE GAP TO CAPACITY UNDER ITERATIVE DECODING. THE DEGREE DISTRIBUTIONS FOR THE ENSEMBLE OF LDPC CODES, AND THE POLYNOMIALS WHICH CORRESPOND TO THE PUNCTURING PATTERNS ARE GIVEN IN [4, TABLE 3].

\begin{tabular}{|c|c|c|c|c|c|}
\hline $\begin{array}{c}\pi^{(0)}(x) \\
\text { (puncturing pattern) }\end{array}$ & $\begin{array}{c}\text { Design } \\
\text { rate }\end{array}$ & $\begin{array}{c}\text { Capacity } \\
\text { limit }\end{array}$ & $\begin{array}{c}\text { Lower bound } \\
\text { (ML decoding) }\end{array}$ & $\begin{array}{l}\text { Iterative (IT) } \\
\text { Decoding }\end{array}$ & $\begin{array}{c}\text { Fractional gap to } \\
\text { capacity (ML vs. IT) }\end{array}$ \\
\hline 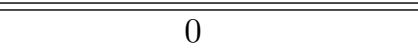 & $\overline{c 0.500}$ & $0.187 \mathrm{~dB}$ & $0.234 \mathrm{~dB}$ & $0.299 \mathrm{~dB}$ & $\geq 41.5 \%$ \\
\hline $\begin{array}{l}0.102040 x+0.06497 x^{2}+ \\
0.06549 x^{5}+0.00331 x^{6}+ \\
0.39377 x^{19}\end{array}$ & 0.555 & $0.450 \mathrm{~dB}$ & $0.473 \mathrm{~dB}$ & $0.599 \mathrm{~dB}$ & $\geq 15.4 \%$ \\
\hline $\begin{array}{l}0.226410 x+0.14149 x^{2}+ \\
0.21268 x^{5}+0.00001 x^{6}+ \\
0.4424 x^{19}\end{array}$ & 0.625 & $0.816 \mathrm{~dB}$ & $0.841 \mathrm{~dB}$ & $1.028 \mathrm{~dB}$ & $\geq 11.9 \%$ \\
\hline $\begin{array}{l}0.348940 x+0.21015 x^{2}+ \\
0.38902 x^{5}+0.00003 x^{6}+ \\
0.48847 x^{19}\end{array}$ & 0.714 & $1.368 \mathrm{~dB}$ & $1.398 \mathrm{~dB}$ & $1.699 \mathrm{~dB}$ & $\geq 8.9 \%$ \\
\hline $\begin{array}{l}0.410320 x+0.24330 x^{2}+ \\
0.48388 x^{5}+0.00004 x^{6}+ \\
0.50541 x^{19}\end{array}$ & 0.769 & $1.777 \mathrm{~dB}$ & $1.811 \mathrm{~dB}$ & $2.215 \mathrm{~dB}$ & $\geq 7.8 \%$ \\
\hline $\begin{array}{l}0.469100 x+0.28408 x^{2}+ \\
0.56178 x^{5}+0.00002 x^{6}+ \\
0.53412 x^{19}\end{array}$ & 0.833 & $2.362 \mathrm{~dB}$ & $2.404 \mathrm{~dB}$ & $3.004 \mathrm{~dB}$ & $\geq 6.6 \%$ \\
\hline $\begin{array}{l}0.533750 x+0.30992 x^{2}+ \\
0.66375 x^{5}+0.00001 x^{6}+ \\
0.54837 x^{19}\end{array}$ & 0.909 & $3.343 \mathrm{~dB}$ & $3.410 \mathrm{~dB}$ & $4.634 \mathrm{~dB}$ & $\geq 5.2 \%$ \\
\hline
\end{tabular}

\section{A. A Lower Bound on the Decoding Complexity for Parallel MBIOS Channels}

Consider a binary linear block code which is represented by a bipartite graph, and assume that the graph serves for the decoding with an iterative algorithm. Following [3] and [9], the decoding complexity under MPI decoding is defined as the number of edges in the graph normalized per information bit. This quantity measures the number of messages which are delivered through the edges of the graph (from left to right and vice versa) during a single iteration. Equivalently, since there is a one-to-one correspondence between a bipartite graph and the parity-check matrix $H$ which represents the code, the decoding complexity is also equal to the number of non-zero elements in $H$ normalized per information bit (i.e., it is equal to the density of the parity-check matrix [14, Definition 2.2]). Hence, the decoding complexity and performance of iteratively decoded binary linear block codes depend on the specific representation of the code by a parity-check matrix. Since the average right degree $\left(a_{\mathrm{R}}\right)$ of a bipartite graph is equal to the number of edges per parity-check equation, then the average right degree and the decoding complexity are two related quantities. Consider an ensemble of LDPC codes whose design rate is $R_{\mathrm{d}}$. It is natural to relate the decoding complexity of the ensemble, say $\chi_{D}$, to its average right degree and design rate, as follows:

$$
\chi_{\mathrm{D}}=\left(\frac{1-R_{\mathrm{d}}}{R_{\mathrm{d}}}\right) a_{\mathrm{R}} .
$$

We note that $a_{\mathrm{R}}$ is fixed for all the codes from an ensemble of LDPC codes with a given pair of degree distributions.

The following lemma is used in the continuation for the derivation of a lower bound on the decoding complexity per iteration under MPI decoding. 
TABLE III

COMPARISON OF THRESHOLDS FOR ENSEMBLES OF IP-LDPC CODES WHERE THE ORIGINAL ENSEMBLE BEFORE PUNCTURING HAS THE DEGREE DISTRIBUTIONS $\lambda(x)=0.414936 x+0.183492 x^{2}+0.013002 x^{3}+0.093081 x^{4}+0.147017 x^{7}+0.148472 x^{24}$ AND $\rho(x)=0.4 x^{2}+0.6 x^{3}$ (SO ITS DESIGN RATE IS $\frac{1}{10}$ ). THE TRANSMISSION OF THESE CODES TAKES PLACE OVER A BINARY-INPUT AWGN CHANNEL. THE TABLE COMPARES VALUES OF $\frac{E_{\mathrm{b}}}{N_{0}}$ REFERRING TO THE CAPACITY LIMIT, THE BOUND GIVEN IN THEOREM 4.2 (WHICH PROVIDES A LOWER BOUND ON THE $\frac{E_{\mathrm{b}}}{N_{0}}$-THRESHOLD UNDER ML DECODING), AND THRESHOLDS UNDER ITERATIVE DECODING. THE FRACTIONAL GAP TO CAPACITY (SEE THE RIGHTMOST COLUMN) MEASURES THE RATIO OF THE GAP TO CAPACITY UNDER ML DECODING AND THE ACHIEVABLE GAP TO CAPACITY UNDER ITERATIVE (SUM-PRODUCT) DECODING. THE DEGREE DISTRIBUTIONS FOR THE ENSEMBLE OF LDPC CODES, AND THE POLYNOMIALS CORRESPONDING TO THE PUNCTURING PATTERNS ARE GIVEN IN [5,

TABLE 5.1].

\begin{tabular}{|c|c|c|c|c|c|}
\hline $\begin{array}{c}\pi^{(0)}(x) \\
\text { (puncturing pattern) }\end{array}$ & $\begin{array}{c}\text { Design } \\
\text { rate }\end{array}$ & $\begin{array}{c}\text { Capacity } \\
\text { limit }\end{array}$ & $\begin{array}{l}\text { Lower bound } \\
\text { (ML decoding) }\end{array}$ & $\begin{array}{l}\text { Iterative (IT) } \\
\text { Decoding }\end{array}$ & $\begin{array}{c}\text { Fractional gap to } \\
\text { capacity (ML vs. IT) }\end{array}$ \\
\hline 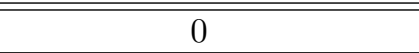 & $\overline{c 0.100}$ & 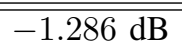 & " -1.248 dB & $-1.028 \mathrm{~dB}$ & $\geq 14.5 \%$ \\
\hline $\begin{array}{l}0.486490 x+0.69715 x^{2}+ \\
0.03287 x^{3}+0.04248 x^{4}+ \\
0.69048 x^{7}+0.45209 x^{24}\end{array}$ & 0.203 & $-0.953 \mathrm{~dB}$ & $-0.917 \mathrm{~dB}$ & $-0.731 \mathrm{~dB}$ & $\geq 16.3 \%$ \\
\hline $\begin{array}{l}0.655580 x+0.83201 x^{2}+ \\
0.48916 x^{3}+0.33917 x^{4}+ \\
0.63990 x^{7}+0.76947 x^{24}\end{array}$ & 0.304 & $-0.605 \mathrm{~dB}$ & $-0.570 \mathrm{~dB}$ & $-0.317 \mathrm{~dB}$ & $\geq 12.0 \%$ \\
\hline $\begin{array}{l}0.745690 x+0.87184 x^{2}+ \\
0.38179 x^{3}+0.48427 x^{4}+ \\
0.74655 x^{7}+0.79130 x^{24}\end{array}$ & 0.406 & $-0.226 \mathrm{~dB}$ & $-0.189 \mathrm{~dB}$ & $+0.029 \mathrm{~dB}$ & $\geq 14.7 \%$ \\
\hline $\begin{array}{l}0.838470 x+0.65105 x^{2}+ \\
0.04527 x^{3}+0.95233 x^{4}+ \\
0.74808 x^{7}+0.80845 x^{24}\end{array}$ & 0.487 & $+0.130 \mathrm{~dB}$ & $+0.171 \mathrm{~dB}$ & $+0.599 \mathrm{~dB}$ & $\geq 8.7 \%$ \\
\hline $\begin{array}{l}0.979320 x+0.46819 x^{2}+ \\
0.71050 x^{3}+0.59816 x^{4}+ \\
0.79485 x^{7}+0.05765 x^{24}\end{array}$ & 0.577 & $+0.556 \mathrm{~dB}$ & $+0.840 \mathrm{~dB}$ & $+1.152 \mathrm{~dB}$ & $\geq 47.7 \%$ \\
\hline $\begin{array}{l}0.895200 x+0.84401 x^{2}+ \\
0.98541 x^{3}+0.42518 x^{4}+ \\
0.92976 x^{7}+0.30225 x^{24}\end{array}$ & 0.663 & $+1.039 \mathrm{~dB}$ & $+1.232 \mathrm{~dB}$ & $+1.806 \mathrm{~dB}$ & $\geq 25.2 \%$ \\
\hline $\begin{array}{l}0.910960 x+0.91573 x^{2}+ \\
0.23288 x^{3}+0.40977 x^{4}+ \\
0.99811 x^{7}+0.15915 x^{24}\end{array}$ & 0.747 & $+1.605 \mathrm{~dB}$ & $+1.958 \mathrm{~dB}$ & $+2.637 \mathrm{~dB}$ & $\geq 34.2 \%$ \\
\hline $\begin{array}{l}0.904130 x+0.96192 x^{2}+ \\
0.35996 x^{3}+0.96980 x^{4}+ \\
0.31757 x^{7}+0.89250 x^{24}\end{array}$ & 0.828 & $+2.303 \mathrm{~dB}$ & $+2.505 \mathrm{~dB}$ & $+3.863 \mathrm{~dB}$ & $\geq 13.0 \%$ \\
\hline
\end{tabular}

Lemma 5.1: Let $\Gamma$ be the right degree distribution of an ensemble of LDPC codes. Then

$$
\Gamma(\alpha) \geq \alpha^{a_{\mathrm{R}}}, \quad \forall \alpha \geq 0 .
$$

Proof: Using the convexity of the function $f(x)=\alpha^{x}$, it follows from Jensen's inequality that for $\alpha \geq 0$

$$
\Gamma(\alpha)=\sum_{i=1}^{\infty} \Gamma_{i} \alpha^{i} \geq \alpha^{\sum_{i=1}^{\infty} i \Gamma_{i}}=\alpha^{a_{\mathrm{R}}} .
$$

Consider a sequence of ensembles of LDPC codes, $\left\{\left(n_{r}, \underline{\lambda}_{r}, \rho\right)\right\}_{r=1}^{\infty}$, whose transmission takes place over a set of $J$ statistically independent parallel MBIOS channels. Let $C_{j}$ and $p_{j}$ be the capacity and the fraction of code bits assigned to the $j^{\text {th }}$ channel, respectively (where $j \in\{1, \ldots, J\}$ ). We define the average capacity of the set of $J$ parallel channels as $\bar{C} \triangleq \sum_{j=1}^{J} p_{j} C_{j}$. For an ensemble of LDPC codes which achieves vanishing bit error probability as the block length tends to infinity, the multiplicative gap (in rate) to capacity is defined as

$$
\varepsilon \triangleq 1-\frac{R_{\mathrm{d}}}{\bar{C}}
$$


We now present a lower bound on the decoding complexity per iteration under MPI decoding for this sequence. The bound is given in terms of the gap to capacity.

Theorem 5.1: Let a sequence of ensembles of binary LDPC codes, $\left\{\left(n_{r}, \underline{\lambda}_{r}, \rho\right)\right\}_{r=1}^{\infty}$, be transmitted over a set of $J$ statistically independent parallel MBIOS channels. Let $C_{j}$ be the capacity of the $j^{\text {th }}$ channel (where $j \in\{1, \ldots, J\}$ ), and denote the average capacity by $\bar{C} \triangleq \sum_{j=1}^{J} p_{j} C_{j}$. If this sequence achieves a fraction $1-\varepsilon$ of $\bar{C}$ with vanishing bit error probability, then the asymptotic decoding complexity under MPI decoding satisfies

$$
\chi_{\mathrm{D}}(\varepsilon) \geq K_{1}+K_{2} \ln \left(\frac{1}{\varepsilon}\right) .
$$

The coefficients $K_{1,2}$ in this lower bound are as follows:

$$
K_{1}=K_{2} \ln \left(\frac{1}{2 \ln 2} \frac{1-\bar{C}}{\bar{C}}\right), \quad K_{2}=-\frac{1-\bar{C}}{\bar{C} \ln \left(\sum_{j=1}^{J} q_{j} g_{j, 1}\right)}
$$

where $g_{j, 1}$ is introduced in (3), and $q_{j}$ is introduced in (25) and is assumed to be positive for all $j \in\{1, \ldots, J\}$. For parallel BECs, the term $\frac{1}{2 \ln 2}$ can be removed from the numerator in the expression of $K_{1}$.

Proof: Substituting (57) in (26) gives

$$
(1-\varepsilon) \bar{C} \leq 1-\frac{1-\bar{C}}{1-\frac{1}{2 \ln 2} \sum_{p=1}^{\infty}\left\{\frac{1}{p(2 p-1)} \Gamma\left(\sum_{j=1}^{J} q_{j} g_{j, p}\right)\right\}} .
$$

Since $g_{j, p}$ in (3) is non-negative for $j \in\{1, \ldots, J\}$ and $p \in \mathbb{N}$, and the function $\Gamma$ is non-negative on $\mathbb{R}^{+}$, then the terms in the infinite sum above are all non-negative. By the truncation of this series where we only take into account its first term (note that this is the largest term in the sum), we obtain a lower bound on the RHS of (60). This implies that

$$
(1-\varepsilon) \bar{C} \leq 1-\frac{1-\bar{C}}{1-\frac{1}{2 \ln 2} \Gamma\left(\sum_{j=1}^{J} q_{j} g_{j, 1}\right)}
$$

Invoking Lemma 5.1 yields that

$$
(1-\varepsilon) \bar{C} \leq 1-\frac{1-\bar{C}}{1-\frac{1}{2 \ln 2}\left(\sum_{j=1}^{J} q_{j} g_{j, 1}\right)^{a_{\mathrm{R}}}} .
$$

The solution of the last inequality for the average right degree $\left(a_{\mathrm{R}}\right)$ gives

$$
\begin{aligned}
a_{\mathrm{R}} \geq-\frac{\ln \left(\frac{1}{2 \ln 2}\left(1+\frac{1-\bar{C}}{\bar{C} \varepsilon}\right)\right)}{\ln \left(\sum_{j=1}^{J} q_{j} g_{j, 1}\right)} \\
>K_{1}^{\prime}+K_{2}^{\prime} \ln \left(\frac{1}{\varepsilon}\right)
\end{aligned}
$$

where the last step follows by dropping the 1 which appeared inside the logarithm at the numerator (this step is valid since the denominator is strictly negative due to the fact that $g_{j, 1} \leq 1$ for all $j$ ), and

$$
K_{1}^{\prime}=-\frac{\ln \left(\frac{1}{2 \ln 2} \frac{1-\bar{C}}{\bar{C}}\right)}{\ln \left(\sum_{j=1}^{J} q_{j} g_{j, 1}\right)}, \quad K_{2}^{\prime}=-\frac{1}{\ln \left(\sum_{j=1}^{J} q_{j} g_{j, 1}\right)} .
$$


Since $R_{\mathrm{d}}<\bar{C}$, it follows that $\chi_{\mathrm{D}}=\frac{1-R_{\mathrm{d}}}{R_{\mathrm{d}}} a_{\mathrm{R}}>\frac{1-\bar{C}}{\bar{C}} a_{\mathrm{R}}$. The proof of the lower bound on the decoding complexity for parallel MBIOS channels follows by multiplying both sides of (61) by $\frac{1-\bar{C}}{\bar{C}}$.

For parallel BECs, we get from (3) that for every $p \in \mathbb{N}$

$$
g_{j, p}=\int_{0}^{\infty} a(l ; j)\left(1+e^{-l}\right) \tanh ^{2 p}\left(\frac{l}{2}\right) \mathrm{d} l=1-\varepsilon_{j}
$$

where $\varepsilon_{j}$ denotes the erasure probability of the $j^{\text {th }}$ BEC. This gives

$$
\begin{aligned}
& \frac{1}{2 \ln 2} \sum_{p=1}^{\infty}\left\{\frac{1}{p(2 p-1)} \Gamma\left(\sum_{j=1}^{J} q_{j} g_{j, p}\right)\right\} \\
& =\frac{1}{2 \ln 2} \sum_{p=1}^{\infty} \frac{1}{p(2 p-1)} \cdot \Gamma\left(\sum_{j=1}^{J} q_{j} g_{j, 1}\right) \\
& =\Gamma\left(\sum_{j=1}^{J} q_{j} g_{j, 1}\right) .
\end{aligned}
$$

Substituting this in (60), gives

$$
(1-\varepsilon) \bar{C} \leq 1-\frac{1-\bar{C}}{1-\Gamma\left(\sum_{j=1}^{J} q_{j} g_{j, 1}\right)} .
$$

The continuation of the proof follows the same steps as the proof for parallel MBIOS channels, and leads to an improved coefficient $K_{1}$ where the factor $\frac{1}{2 \ln 2}$ in the numerator of $K_{1}$ for general MBIOS channels (see (59)) is replaced by 1 .

We proceed the analysis by the derivation of lower bounds on the decoding complexity of sequences of ensembles of punctured LDPC codes where it is assumed that these sequences achieve vanishing bit error probability; similarly to Theorem 5.1, the lower bounds are expressed in terms of the multiplicative gap (in rate) to capacity.

\section{B. Lower Bounds on the Decoding Complexity for Punctured LDPC Codes}

As discussed in the previous section, transmission of punctured codes can be interpreted as a special case of transmitting the original (un-punctured) codes over a set of parallel channels where these component channels are formed by a mixture of the communication channel and BECs whose erasure probabilities are the puncturing rates of the different subsets of code bits. Hence, the bounds on the decoding complexity of punctured codes can be derived as special cases of the bound given in Theorem 5.1. For the sake of brevity, we derive these bounds by using the upper bounds on the achievable rates of punctured LDPC codes as given in Theorem 4.1 (for random puncturing) and Theorem 4.2 (for intentional puncturing). Note that the derivation of these two theorems relies on Theorem 3.1 (as shown in Fig. 1 on p. 27).

Consider an ensemble of LDPC codes of length $n$ and design rate $R_{\mathrm{d}}^{\prime}$, and let the code bits be partitioned into $J$ disjoint sets where the $j^{\text {th }}$ set contains a fraction $p_{j}$ of these bits $(j \in\{1, \ldots, J\})$. Assume that the bits in the $j^{\text {th }}$ set are randomly punctured at rate $\pi_{j}$, and let the punctured codes be transmitted over an MBIOS channel whose capacity is $C$. As shown in the previous section, this is equivalent to transmitting the original (un-punctured) codes over a set of $J$ parallel channels, where the $j^{\text {th }}$ set of code bits is transmitted over a channel whose capacity is $C_{j}=\left(1-\pi_{j}\right) C$. The average capacity of this set of $J$ parallel channels is therefore given by

$$
\bar{C}=\sum_{j=1}^{J} p_{j}\left(1-\pi_{j}\right) C=\left(1-\sum_{j=1}^{J} p_{j} \pi_{j}\right) C=(1-\gamma) C
$$

where $\gamma \triangleq \sum_{j=1}^{J} p_{j} \pi_{j}$ is the overall puncturing rate. Denote the design rate of the punctured codes by $R_{\mathrm{d}} \triangleq \frac{R_{\mathrm{d}}^{\prime}}{1-\gamma}$ (see Definition 4.1 on p. 14), then it follows that the multiplicative gap to capacity of the punctured codes is given by

$$
\varepsilon=1-\frac{R_{\mathrm{d}}}{C}=1-\frac{R_{\mathrm{d}}^{\prime}}{\bar{C}} .
$$


For punctured codes, the iterative decoder is based on the bipartite graph of the 'mother code' where the channel input to the variable nodes which correspond to the punctured code bits is defined to be 0 . Hence, the decoding complexity of the punctured ensemble under MPI decoding is identical to the decoding complexity of the original ensemble (before puncturing), and is given by

$$
\begin{aligned}
\chi_{\mathrm{D}} & =\left(\frac{1-R_{\mathrm{d}}^{\prime}}{R_{\mathrm{d}}^{\prime}}\right) a_{\mathrm{R}} \\
& =\left(\frac{1-(1-\gamma) R_{\mathrm{d}}}{(1-\gamma) R_{\mathrm{d}}}\right) a_{\mathrm{R}} .
\end{aligned}
$$

In the following, we derive a lower bound on the decoding complexity of a sequence of ensembles of RP-LDPC codes.

Theorem 5.2: Let $\left\{\left(n_{r}, \lambda, \rho\right)\right\}_{r=1}^{\infty}$ be a sequence of ensembles of binary LDPC codes whose block length $\left(n_{r}\right)$ tends to infinity as $r \rightarrow \infty$. Assume that a sequence of ensembles of RP-LDPC codes is constructed in the following way: for each code from an ensemble of the original sequence, a subset of $\alpha n_{r}$ code bits is a-priori selected, and these bits are randomly punctured at a fixed rate $\left(P_{\mathrm{pct}}\right)$. Assume that the punctured codes are transmitted over an MBIOS channel with capacity $C$, and that as $r$ tends to infinity, the sequence of ensembles of punctured codes achieves a fraction $1-\varepsilon$ of the capacity with vanishing bit error probability. Then in probability 1 w.r.t. the random puncturing patterns, the decoding complexity of this sequence under MPI decoding satisfies

$$
\chi_{\mathrm{D}}(\varepsilon) \geq K_{1}+K_{2} \ln \left(\frac{1}{\varepsilon}\right) .
$$

The coefficients $K_{1,2}$ in this lower bound are as follows:

$$
\begin{aligned}
& K_{1}=K_{2} \ln \left(\frac{1}{2 \ln 2} \frac{1-\bar{C}}{\bar{C}}\right) \\
& K_{2}=-\frac{1-\bar{C}}{\bar{C} \ln \left(\left(1-P_{\mathrm{pct}}+\xi\right) g_{1}\right)}
\end{aligned}
$$

where $g_{1}$ is introduced in (40), $\xi$ is introduced in (42), and $\bar{C} \triangleq\left(1-\alpha P_{\mathrm{pct}}\right) C$. For the particular case of a BEC, the term $\frac{1}{2 \ln 2}$ can be dropped, thus improving the tightness of the additive term $\left(K_{1}\right)$ in the lower bound.

Proof: Since a subset of the code bits of size $\alpha n_{r}$ is randomly punctured at rate $P_{\text {pct }}$, then the average puncturing rate is given by $\gamma=\alpha P_{\text {pct }}$. Hence, Eq. (62) yields that $\bar{C}=\left(1-\alpha P_{\mathrm{pct}}\right) C$. By multiplying both sides of (41) by $1-\alpha P_{\text {pct }}$ and getting from (63) that $R_{\mathrm{d}}=(1-\varepsilon) C$, we obtain

$$
(1-\varepsilon) \bar{C} \leq 1-\frac{1-\bar{C}}{1-\frac{1}{2 \ln 2} \sum_{p=1}^{\infty} \frac{\Gamma\left(\left(1-P_{\mathrm{pct}}+\xi\right) g_{p}\right)}{p(2 p-1)} .}
$$

Following the same steps as in the proof of Theorem 5.1, we get a lower bound on the average right degree of the bipartite graph which corresponds to the pair of degree distributions $(\lambda, \rho)$. This lower bound is of the form

$$
a_{\mathrm{R}}>K_{1}^{\prime}+K_{2}^{\prime} \ln \left(\frac{1}{\varepsilon}\right)
$$

where

$$
\begin{aligned}
& K_{1}^{\prime}=K_{2}^{\prime} \ln \left(\frac{1}{2 \ln 2} \frac{1-\bar{C}}{\bar{C}}\right) \\
& K_{2}^{\prime}=-\frac{1}{\ln \left(\left(1-P_{\mathrm{pct}}+\xi\right) g_{1}\right)} .
\end{aligned}
$$

Note that $K_{2}^{\prime}$ is positive; this follows from (42), which yields that $\xi<(1-\alpha) P_{\text {pct }}$ (due to the fact that the integral of $\lambda$ over the interval $[0,1]$ is upper bounded by $\frac{1}{2}$ ). This assures that as the gap (in rate) to capacity vanishes, the lower bound on $a_{\mathrm{R}}$ scales like the logarithm of the inverse of this gap. 
From (63), we get that $R_{\mathrm{d}}^{\prime}=(1-\varepsilon) \bar{C}<\bar{C}$, and therefore $\chi_{\mathrm{D}}=\frac{1-R_{\mathrm{d}}^{\prime}}{R_{\mathrm{d}}^{\prime}} a_{\mathrm{R}}>\frac{1-\bar{C}}{\bar{C}} a_{\mathrm{R}}$. The proof of the lower bound on the decoding complexity is completed by multiplying both sides of (67) by $\frac{1-\bar{C}}{\bar{C}}$. In the particular case where the communication channel is a BEC, following the same concept as in the proof of Theorem 5.1 leads to the improved coefficient $K_{1}$ where the term $\frac{1}{2 \ln 2}$ is dropped.

The upper bound on the decoding complexity for sequences of ensembles of IP-LDPC codes is also given in terms of the gap between the rate of the punctured rate and the channel capacity.

Theorem 5.3: Let $\left\{\left(n_{r}, \lambda, \rho, \pi^{(0)}\right)\right\}_{r=1}^{\infty}$ be a sequence of ensembles of binary IP-LDPC codes transmitted over an MBIOS channel whose channel capacity is $C$. If this sequence achieves a fraction $1-\varepsilon$ of the capacity with vanishing bit error probability, then in probability 1 w.r.t. the random puncturing patterns, the decoding complexity of this sequence under MPI decoding satisfies

$$
\chi_{\mathrm{D}}(\varepsilon) \geq K_{1}+K_{2} \ln \left(\frac{1}{\varepsilon}\right) .
$$

The coefficients $K_{1,2}$ in this lower bound are as follows:

$$
\begin{aligned}
& K_{1}=K_{2} \ln \left(\frac{1}{2 \ln 2} \frac{1-\bar{C}}{\bar{C}}\right) \\
& K_{2}=-\frac{1-\bar{C}}{\bar{C} \ln \left(\left(1-\sum_{j=1}^{\infty} \lambda_{j} \pi_{j}\right) g_{1}\right)}
\end{aligned}
$$

where $g_{1}$ is introduced in (40), and $\bar{C} \triangleq\left(1-\sum_{j=1}^{\infty} \Lambda_{j} \pi_{j}\right) C$. For the particular case of a BEC, the term $\frac{1}{2 \ln 2}$ can be dropped, thus improving the tightness of the additive term $\left(K_{1}\right)$ in the lower bound.

Proof: The proof follows from the same concepts as the proof of Theorem 5.2, but is based on (50) instead of (41). Note that $K_{2}$, which reflects the logarithmic growth rate of the lower bound in (68), is always positive; this follows from (69) and due to the fact that from (40), $g_{1} \leq 1$, and also $0<1-\sum_{j=1}^{\infty} \lambda_{j} \pi_{j} \leq 1$.

\section{Re-Derivation of Reported Lower Bounds on the Decoding Complexity}

In [9, Theorems 3 and 4], Pfister et al. introduced lower bounds on the decoding complexity of punctured codes under iterative decoding. The bounds were derived for the case where a subset of linearly independent code bits whose size is equal to the code dimension are randomly punctured at a fixed rate $\left(P_{\mathrm{pct}}\right)$, and the transmission of the codes takes place over an MBIOS channel. In particular, this scenario corresponds to RP-LDPC codes (see Section IV-B) where we choose a subset of the code bits to be randomly punctured at rate $P_{\text {pct }}$; under the assumption in [9, Theorems 3 and 4], the fraction $(\alpha)$ of the code bits which are randomly punctured is equal to the code rate. In the appendix, we show that for RP-LDPC codes, the lower bounds on the decoding complexity given in [9, Theorems 3 and 4] follow from a looser version of the bound in Theorem 5.2.

\section{Summary AND OUTLOOK}

Theorem 3.1, which is one of the main results in this paper, provides an upper bound on the asymptotic rate of a sequence of ensembles of binary low-density parity-check (LDPC) codes which achieves vanishing bit error probability. We assume that the communication takes place over a set of parallel memoryless binary-input outputsymmetric (MBIOS) channels. The derivation of Theorem 3.1 relies on upper and lower bounds on the conditional entropy of the transmitted codeword given the received sequence at the output of the parallel channels (see Section II), and it holds under optimal maximum-likelihood (ML) decoding (or an arbitrary sub-optimal decoding algorithm). This theorem enables the derivation of a lower bound on the decoding complexity (per iteration) of ensembles of binary LDPC codes under message-passing iterative decoding when the transmission of the codes takes place over a set of parallel MBIOS channels. The latter bound is given in terms of the gap between the rate of these codes for which reliable communication is achievable and the channel capacity. Similarly to a lower bound on the decoding complexity of ensembles of LDPC codes for a single MBIOS channel [14], the lower bound on the decoding complexity which is derived for parallel channels grows like the log of the inverse of the gap (in rate) to capacity. 


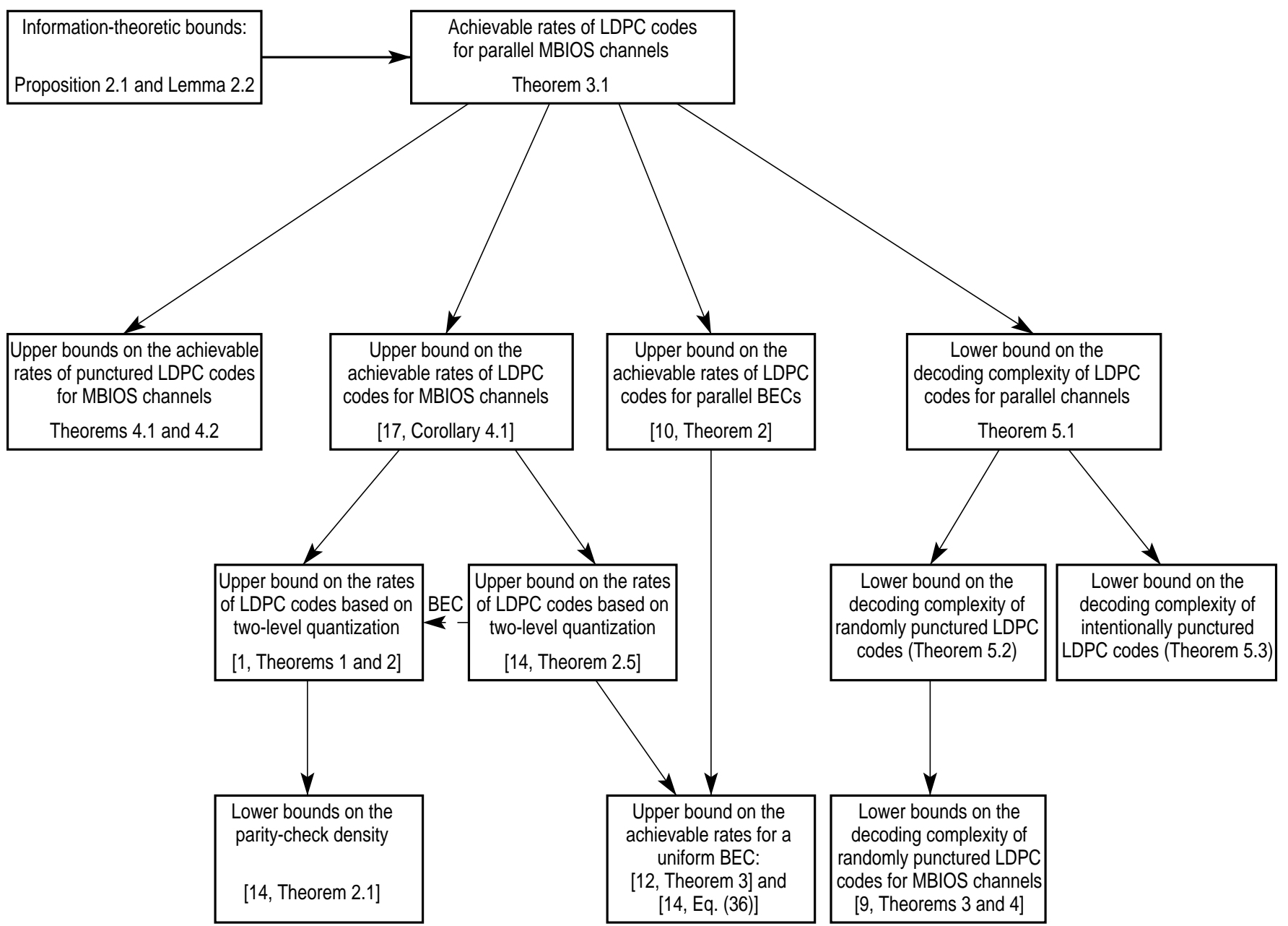

Fig. 1. An interconnections diagram among the bounds in this paper and some previously reported bounds which follow as special cases.

Theorem 3.1 can be used for various applications which form particular cases of communication over parallel channels, e.g., intentionally punctured LDPC codes [4], non-uniformly error protected LDPC codes [10], and LDPCcoded modulation (see e.g., [6], [16]). In Section IV, we rely on Theorem 3.1 for the derivation of upper bounds on the achievable rates under ML decoding of (randomly and intentionally) punctured LDPC codes whose transmission takes place over an arbitrary MBIOS channel. It is exemplified numerically that for various good ensembles of intentionally punctured LDPC codes, the asymptotic loss in performance due to the code structure is non-negligible as compared to the corresponding loss due to the sub-optimality of iterative decoding (as compared to optimal ML decoding). Looser versions of the bounds derived in this paper for punctured LDPC codes suggest a simplified re-derivation of previously reported bounds on the decoding complexity of randomly punctured LDPC codes (see [9, Theorems 3 and 4] and the appendix).

Interconnections between the information-theoretic bounds introduced in this paper and some previously reported results are shown in Fig. 1; it is shown in this figure that results from [1], [9], [10], [12], [14], [17], which correspond to the achievable rates and decoding complexity of LDPC codes (with and without puncturing), follow as special cases of the bounds introduced in this paper.

\section{Acknowledgment}

I. Sason wishes to acknowledge Henry Pfister and Ruediger Urbanke for stimulating discussions on puncturing theorems during the preparation of the work in [9]. The authors are grateful to one of the anonymous reviewers 
who pointed out Remarks 3.1 and 4.2 in this paper. The work of I. Sason was supported by the Taub and Shalom Foundations.

\section{APPENDIX: RE-DERIVATION OF [9, THEOREMS 3 AND 4]}

In the following, we start with the re-derivation of [9, Theorem 4] for general MBIOS channels, and then rederive the refined bound in [9, Theorem 3] for a BEC. For the re-derivation of [9, Theorems 3 and 4] we rely on Theorem 5.2 whose derivation is based on Theorem 4.1. Hence, we first loosen the upper bound on the achievable rates given in (41), and then re-derive [9, Theorem 4] as a consequence of this looser version. The loosening of (41) is done by replacing the positive parameter $\xi$ introduced in (42) by zero, and then using the lower bound on $\Gamma$ from Lemma 5.1. This gives

$$
\begin{aligned}
R_{\mathrm{d}} \leq & \frac{1}{1-\alpha P_{\mathrm{pct}}} \\
& \cdot\left(1-\frac{1-\left(1-\alpha P_{\mathrm{pct}}\right) C}{\left.1-\frac{1}{2 \ln 2} \sum_{p=1}^{\infty}\left\{\frac{1}{p(2 p-1)}\left(\left(1-P_{\mathrm{pct}}\right) g_{p}\right)^{a_{\mathrm{R}}}\right\}\right)}\right)
\end{aligned}
$$

Truncating the infinite series in the RHS of (A.1) by only taking its first term which corresponds to $p=1$ further loosens the upper bound on the achievable rates, and gives

$$
R_{\mathrm{d}} \leq \frac{1}{1-\alpha P_{\mathrm{pct}}}\left(1-\frac{1-\left(1-\alpha P_{\mathrm{pct}}\right) C}{1-\frac{1}{2 \ln 2}\left(\left(1-P_{\mathrm{pct}}\right) g_{1}\right)^{a_{\mathrm{R}}}}\right)
$$

From (63), we get the inequality

$$
(1-\varepsilon)\left(1-\alpha P_{\mathrm{pct}}\right) C \leq 1-\frac{1-\left(1-\alpha P_{\mathrm{pct}}\right) C}{1-\frac{1}{2 \ln 2}\left(\left(1-P_{\mathrm{pct}}\right) g_{1}\right)^{a_{\mathrm{R}}}}
$$

which after straightforward algebra gives

$$
1+\frac{1-\left(1-\alpha P_{\mathrm{pct}}\right) C}{\varepsilon C\left(1-\alpha P_{\mathrm{pct}}\right)} \leq 2 \ln 2\left(\frac{1}{\left(1-P_{\mathrm{pct}}\right) g_{1}}\right)^{a_{\mathrm{R}}} .
$$

We proceed by giving a simple lower bound on $g_{1}$ in (40).

Lemma A.1: The inequality $g_{1} \geq(1-2 w)^{2}$ holds for any MBIOS channel where

$$
w \triangleq P_{\mathrm{e}}(a)=\frac{1}{2} \operatorname{Pr}(L=0)+\int_{-\infty}^{0^{-}} a(l) \mathrm{d} l
$$

designates the uncoded bit error probability of the MBIOS channel given the channel input is zero. 
Proof: Based on the symmetry property where $a(l)=e^{l} a(-l)$ and Jensen's inequality, we get

$$
\begin{aligned}
g_{1} & =\int_{0}^{\infty} a(l)\left(1+e^{-l}\right) \tanh ^{2}\left(\frac{l}{2}\right) \mathrm{d} l \\
& =\int_{-\infty}^{\infty} a(l) \tanh ^{2}\left(\frac{l}{2}\right) \mathrm{d} l \\
& \geq\left(\int_{-\infty}^{\infty} a(l) \tanh \left(\frac{l}{2}\right) \mathrm{d} l\right)^{2} \\
& =\left(\int_{0}^{\infty} a(l)\left(1+e^{-l}\right) \tanh \left(\frac{l}{2}\right) \mathrm{d} l\right)^{2} \\
& =\left(\int_{0}^{\infty} a(l)\left(1-e^{-l}\right) \mathrm{d} l\right)^{2} \\
& =\left(\int_{0^{+}}^{\infty}(a(l)-a(-l)) \mathrm{d} l\right)^{2} \\
& =\left(1-\operatorname{Pr}(L=0)-2 \int_{-\infty}^{0^{-}} a(l) \mathrm{d} l\right)^{2} \\
& =(1-2 w)^{2} .
\end{aligned}
$$

Replacing $g_{1}$ in the RHS of (A.3) by its lower bound from Lemma A.1 gives

$$
\begin{aligned}
1+\frac{1-\left(1-\alpha P_{\mathrm{pct}}\right) C}{\varepsilon C\left(1-\alpha P_{\mathrm{pct}}\right)} & \leq 2 \ln 2\left(\frac{1}{\left(1-P_{\mathrm{pct}}\right)(1-2 w)^{2}}\right)^{a_{\mathrm{R}}} \\
& \leq 2 \ln 2\left(\frac{1}{\left(1-P_{\mathrm{pct}}\right)(1-2 w)}\right)^{2 a_{\mathrm{R}}} .
\end{aligned}
$$

Solving the last inequality for $a_{\mathrm{R}}$ gives

$$
a_{\mathrm{R}} \geq \frac{\ln \left(\frac{1}{2 \ln 2}\left(1+\frac{1-\left(1-\alpha P_{\mathrm{pct}}\right) C}{\varepsilon C\left(1-\alpha P_{\mathrm{pct}}\right)}\right)\right)}{2 \ln \left(\frac{1}{\left(1-P_{\mathrm{pct}}\right)(1-2 w)}\right)} .
$$

Based on the equality (64) which relates the complexity under iterative decoding to the average right degree $\left(a_{\mathrm{R}}\right)$ and also since $R_{\mathrm{d}}<C$, we get from the last inequality

$$
\chi_{\mathrm{D}}(\varepsilon) \geq \frac{1-C}{2 C} \frac{\ln \left(\frac{1}{2 \ln 2}\left(1+\frac{1-\left(1-\alpha P_{\mathrm{pct}}\right) C}{\varepsilon C\left(1-\alpha P_{\mathrm{pct}}\right)}\right)\right)}{\ln \left(\frac{1}{\left(1-P_{\mathrm{pct}}\right)(1-2 w)}\right)} .
$$

Note that in the setting of [9, Theorem 4], $\alpha=R_{\mathrm{d}}^{\prime}$. This gives the equality $\alpha=(1-\varepsilon) \bar{C}=(1-\varepsilon)\left(1-\alpha P_{\mathrm{pct}}\right) C$ whose solution is

$$
\alpha=\frac{(1-\varepsilon) C}{1+(1-\varepsilon) C P_{\mathrm{pct}}} .
$$

Finally, the substitution of $\alpha$ in (A.5) into the RHS of (A.4) gives

$$
\begin{aligned}
\chi_{\mathrm{D}}(\varepsilon) & \geq \frac{1-C}{2 C} \frac{\ln \left(\frac{1}{2 \ln 2}\left(1+\frac{1-\left(1-P_{\mathrm{pct}}\right) C-\varepsilon C P_{\mathrm{pct}}}{\varepsilon C}\right)\right)}{\ln \left(\frac{1}{\left(1-P_{\mathrm{pct}}\right)(1-2 w)}\right)} \\
& \geq \frac{1-C}{2 C} \frac{\ln \left(\frac{1}{\varepsilon} \frac{1-\left(1-P_{\mathrm{pct}}\right) C}{2 C \ln 2}\right)}{\ln \left(\frac{1}{\left(1-P_{\mathrm{pct}}\right)(1-2 w)}\right)}
\end{aligned}
$$


which coincides with [9, Theorem 4] for a sequence of ensembles of randomly punctured LDPC codes.

For the derivation of the refined bound for the BEC which is given in [9, Theorem 3], we start from (A.1). The refinement of the latter bound is due to the fact that for the BEC, $g_{p}$ in (40) is independent of $p$, and satisfies $g_{p}=1-P_{\mathrm{BEC}}$ where $P_{\mathrm{BEC}}$ designates the erasure probability of the BEC. From (A.1), we get the following upper bound on the achievable rates:

$$
R_{\mathrm{d}} \leq \frac{1}{1-\alpha P_{\mathrm{pct}}}\left(1-\frac{1-\left(1-\alpha P_{\mathrm{pct}}\right) C}{1-\left(\left(1-P_{\mathrm{pct}}\right)\left(1-P_{\mathrm{BEC}}\right)\right)^{a_{\mathrm{R}}}}\right)
$$

which follows from the equality $\sum_{p=1}^{\infty} \frac{1}{2 p(2 p-1)}=\ln 2$. Substituting $R_{\mathrm{d}}=(1-\varepsilon)\left(1-P_{\mathrm{BEC}}\right)$ and the $\alpha$ in (A.5) gives a lower bound on $a_{\mathrm{R}}$. Finally, the lower bound in [9, Theorem 3] follows from the resulting lower bound on $a_{\mathrm{R}}$ and the inequality $\chi_{D}(\varepsilon) \geq \frac{1-C}{C} a_{\mathrm{R}}$.

\section{REFERENCES}

[1] D. Burshtein, M. Krivelevich, S. Litsyn and G. Miller, "Upper bounds on the rate of LDPC codes," IEEE Trans. on Information Theory, vol. 48, no. 9, pp. 2437-2449, September 2002.

[2] C. H. Hsu and A. Anastasopoulos, "Capacity-achieving LDPC codes through puncturing," Proceedings 2005 IEEE International Conference on Wireless Networks, Communications and Mobile Computing (WirelessCom 2005), Mauii, Hawaii, USA, June 13-16, 2005.

[3] Y. Liu, J. Hou and V. K. N. Lau, “Complexity bounds of LDPC codes for parallel channels,” Proceedings Forty-Second Annual Allerton Conference on Communication, Control and Computing, pp. 1705-1713, Urbana-Champaign, IL, USA, September 29-October 1, 2004.

[4] J. Ha and S. W. McLaughlin, "Rate-compatible puncturing of low-density parity-check codes," IEEE Trans. on Information Theory, vol. 50, no. 11, pp. 2824-2836, November 2004.

[5] J. Ha, Low-Density Parity-Check Codes with Erasures and Puncturing, Ph.D. dissertation, Georgia Institute of Technology, November 2003.

[6] J. Hou, P. H. Siegel, L. B. Milstein and H. D. Pfister, "Capacity-approaching bandwidth-efficient coded modulation schemes based on low-density parity-check codes," IEEE Trans. on Information Theory, vol. 49, no. 9, pp. 2141-2155, September 2003.

[7] R. Liu , P. Spasojevic and E. Soljanin, "Reliable channel regions for good codes transmitted over parallel channels," IEEE Trans. on Information Theory, vol. 52, pp. 1405-1424, April 2006.

[8] C. Measson, A. Montanari and R. Urbanke, "Maxwell construction: the hidden bridge between iterative and maximum a posteriori decoding," accepted to IEEE Trans. on Information Theory, 2006. [Online]. Available: http: / / www. arxiv.org/abs/cs. IT/0506083.

[9] H. D. Pfister, I. Sason and R. Urbanke, "Capacity-achieving ensembles for the binary erasure channel with bounded complexity," IEEE Trans. on Information Theory, vol. 51, no. 7, pp. 2352-2379, July 2005.

[10] H. Pishro-Nik, N. Rahnavard and F. Fekri, "Nonuniform error correction using low-density parity-check codes," IEEE Trans. on Information Theory, vol. 51, no. 7, pp. 2702-2714, July 2005.

[11] H. Pishro-Nik and F. Fekri, "Results on punctured LDPC codes," Proceedings 2004 IEEE Information Theory Workshop (ITW 2004), pp. 215-219, San Antonio, Texas, USA, October 24-29, 2004.

[12] H. Pishro-Nik and F. Fekri, "On decoding of low-density parity-check codes over the binary erasure channel," IEEE Trans. on Information Theory, vol. 50, pp. 439-454, March 2004.

[13] T. Richardson and R. Urbanke, Modern Coding Theory, to be published, Cambridge Press, preprint. [Online]. Available: http: //lthcwww.epfl.ch/mct/index.php.

[14] I. Sason and R. Urbanke, "Parity-check density versus performance of binary linear block codes over memoryless symmetric channels," IEEE Trans. on Information Theory, vol. 49, no. 7, pp. 1611-1635, July 2003.

[15] I. Sason and I. Goldenberg, "Coding for parallel channels: Gallager bounds and applications to repeat-accumulate codes," Proceedings of the 24th IEEE Convention of Electrical and Electronics Engineers in Israel, pp. 344-348, Eilat, Israel, November 15-17, 2006.

[16] T. Wadayama, "A coded modulation scheme based on low-density parity-check codes," IEICE Transactions on Fundamentals, vol. E84A, no. 10, pp. 2523-2527, October 2001.

[17] G. Wiechman and I. Sason, "Parity-check density versus performance of binary linear block codes: new bounds and applications," to appear in the IEEE Trans. on Information Theory, vol. 53, February 2007. 